\title{
Astrocyte Contributions to Flow/Pressure-Evoked Parenchymal Arteriole Vasoconstriction
}

\author{
Ki Jung Kim, ${ }^{1}$ Jennifer A. Iddings, ${ }^{1}$ Javier E. Stern, ${ }^{1}$ Víctor M. Blanco, ${ }^{2}$ Deborah Croom, ${ }^{1}$ Sergei A. Kirov, ${ }^{1}$ \\ and Jessica A. Filosa ${ }^{1}$ \\ ${ }^{1}$ Georgia Regents University, Augusta, Georgia 30912, and 2University of Cincinnati, Cincinnati, Ohio 45221
}

\begin{abstract}
Basal and activity-dependent cerebral blood flow changes are coordinated by the action of critical processes, including cerebral autoregulation, endothelial-mediated signaling, and neurovascular coupling. The goal of our study was to determine whether astrocytes contribute to the regulation of parenchymal arteriole (PA) tone in response to hemodynamic stimuli (pressure/flow). Cortical PA vascular responses and astrocytic $\mathrm{Ca}^{2+}$ dynamics were measured using an in vitro rat/mouse brain slice model of perfused/pressurized PAs; studies were supplemented with in vivo astrocytic $\mathrm{Ca}^{2+}$ imaging. In vitro, astrocytes responded to PA flow/pressure increases with an increase in intracellular $\mathrm{Ca}^{2+}$. Astrocytic $\mathrm{Ca}^{2+}$ responses were corroborated in vivo, where acute systemic phenylephrine-induced increases in blood pressure evoked a significant increase in astrocytic $\mathrm{Ca}^{2+}$. In vitro, flow/pressure-evoked vasoconstriction was blunted when the astrocytic syncytium was loaded with BAPTA (chelating intracellular $\mathrm{Ca}^{2+}$ ) and enhanced when high $\mathrm{Ca}^{2+}$ or ATP were introduced to the astrocytic syncytium. Bath application of either the TRPV4 channel blocker HC067047 or purinergic receptor antagonist suramin blunted flow/pressure-evoked vasoconstriction, whereas $\mathrm{K}^{+}$and 20-HETE signaling blockade showed no effect. Importantly, we found TRPV4 channel expression to be restricted to astrocytes and not the endothelium of PA. We present evidence for a novel role of astrocytes in PA flow/pressure-evoked vasoconstriction. Our data suggest that astrocytic TRPV4 channels are key molecular sensors of hemodynamic stimuli and that a purinergic, glial-derived signal contributes to flow/pressure-induced adjustments in PA tone. Together our results support bidirectional signaling within the neurovascular unit and astrocytes as key modulators of PA tone.
\end{abstract}

Key words: astrocyte; calcium; myogenic; neurovascular; parenchymal arteriole; vascular tone

\section{Introduction}

Activity-dependent cerebral blood flow (CBF) increases are mainly governed by neurovascular coupling (NVC) mechanisms (Attwell et al., 2010; Cauli and Hamel, 2010), whereas basal/resting $\mathrm{CBF}$ is coordinated through multiple processes, including cerebral autoregulation (CA) and endothelial-mediated signaling. Notably, less is understood about the mechanisms underlying basal CBF, which is important when assessing NVC-mediated responses and because reduced $\mathrm{CBF}$ and impaired CA are observed in pathological conditions contributing to cognitive decline (Kennelly et al., 2009). CA, mediated by the integration of myogenic, neurogenic, and metabolic responses, ensures constant $\mathrm{CBF}$ throughout a broad range of systemic blood pressures

Received 0ct. 29, 2014; revised March 27, 2015; accepted April 24, 2015.

Author contributions: K.J.K., J.A.I., J.E.S., and J.A.F. designed research; K.J.K., J.A.I., J.E.S., V.M.B., D.C., S.A.K., and

J.A.F. performed research; K.J.K., J.A.I., J.E.S., and J.A.F. analyzed data; K.J.K., J.A.I., J.E.S., and J.A.F. wrote the paper.

This work was supported by the National Institutes of Health National Heart, Lung and Blood Institute Grant R01 HL089067-02 to J.A.F., the American Heart Association Grant 11PRE7400037 to J.A.I., National Institutes of Health Grant HL112225 to J.E.S., and National Institutes of Health Grant NS083858 and American Heart Association Grant 12GRNT16570006 to S.A.K. We thank Drs. Michael Brands, Helena Morrison, and Jeremy Sword from Georgia Regents University for their assistance on the in vivo experiments. We also thank Dr. Wolfgang Liedtke from the Center for Translational Neuroscience, Duke University, Durham, North Carolina, for providing us with the TRPV4 ${ }^{-1-}$ mice as well as helpful comments on the manuscript.

The authors declare no competing financial interests.

Correspondence should be addressed to Jessica A. Filosa, Department of Physiology, Georgia Regents University, Augusta, GA 30912. E-mail: jillosa@gru.edu.

DOI:10.1523/JNEUROSCI.4486-14.2015

Copyright $\odot 2015$ the authors $\quad 0270-6474 / 15 / 358245-13 \$ 15.00 / 0$
(50-150 mmHg; Cipolla et al., 2009). The myogenic component is the best understood (Faraci et al., 1989; Harder et al., 2011; Koller and Toth, 2012); it involves cellular mechanisms that regulate vascular smooth muscle cell (VSMC) $\mathrm{Ca}^{2+}$ (Brayden et al., 2008) resulting in vasoconstriction or vasodilation in response to increases or decreases in pressure, respectively. However, most studies have focused on pial arteriole myogenic responses, with only a few (Nakahata et al., 2006; Chan et al., 2013) addressing that of parenchymal arterioles (PAs).

Distinct differences between pial arterioles and PAs call for a closer look at their biomechanical properties. As opposed to pial arterioles, which have multiple VSMC layers, PAs have a single VSMC layer. Moreover, a layer of astrocytic endfeet covers the abluminal side of PAs. Because astrocytes possess comparable signaling modalities as those described in endothelial cells (ECs; e.g., arachidonic acid metabolites, nitric oxide, epoxyeicosatrienoic acids, adenosine, and $\mathrm{K}^{+}$; Félétou et al., 2011, 2012), they may constitute an additional regulatory system for the control of PA tone. Specifically, whether astrocytes respond to and/or contribute to the regulation of resting/basal PA tone is not known. We hypothesized that astrocytes act as bidirectional sensors and transducers of brain perfusion, contributing to basal hemodynamic-induced adjustments in PA tone.

TRPV4 channels $\left(\mathrm{Ca}^{2+}\right.$-permeable nonselective cation channels) contribute to the regulation of vascular tone (Earley et al., 2009). In the cerebral cortex, TRPV4 channels are localized on 
astrocytic membranes including endfeet (Benfenati et al., 2007). TPRV4 channels can be activated by osmotic, physical (cell swelling, warmth, mechanical), and chemical (endocannabinoids, arachidonic acid metabolites, $4-\alpha$-phorbol esters) stimuli (Vriens et al., 2004; Plant and Strotmann, 2007), making them attractive candidates to be influenced by hemodynamic variables (e.g., pressure/flow) and contributors to $\mathrm{Ca}^{2+}$-dependent gliovascular signals. TRPV4-dependent astrocyte $\mathrm{Ca}^{2+}$ elevations during NVC were recently reported (Dunn et al., 2013). Thus, we tested whether astrocytic TRPV4 channels contribute to hemodynamic-evoked changes in PA tone.

Using an in vitro/in vivo approach where we systematically regulate flow in PAs while we concurrently monitor PA diameter or $\mathrm{Ca}^{2+}$ in VSMCs and astrocytes, we provide evidence for astrocytic contribution to the regulation of PA tone. Local and systemic hemodynamic stimuli engage astrocytes via a TRPV4-dependent pathway to modulate the magnitude and duration of flow/pressure-evoked PA constrictions. We propose that a purinergic, glial-derived signal contributes to this response. These data provide novel evidence for an active role of astrocytes in flow/pressure-evoked PA constriction and bidirectional communication in the neurovascular unit.

\section{Materials and Methods}

Animals. All in vitro studies with the exception of those reported in Figure 6 were conducted in male juvenile [postnatal day (P) 21-P28] Wistar rats. In vitro experiments shown in Figure 6 were performed in 6-10week-old male TRPV4 ${ }^{-/-}$and TRPV4 ${ }^{+/+}$mice bred on a C57BL6 background kindly provided by Dr. Wolfgang Liedtke, Duke University. Experiments were performed following protocols approved by the animal care and use committee of Georgia Regents University. Rats/mice were housed in a room maintained at $20-22^{\circ} \mathrm{C}$ with a $12 \mathrm{~h} \mathrm{light/dark}$ cycle and given ad libitum access to food and water.

Brain slice preparation. Cortical brain slices were prepared from male Wistar rats or mice. Following anesthesia with sodium pentobarbital, the brain was removed and cut into $250-300-\mu \mathrm{m}$-thick coronal slices using a vibratome (Leica VT 1200S, Leica Microsystems) in cold artificial CSF (aCSF) containing the following: $3 \mathrm{mmol} / \mathrm{L} \mathrm{KCl}, 120 \mathrm{mmol} / \mathrm{L} \mathrm{NaCl}, 1$ $\mathrm{mmol} / \mathrm{L} \mathrm{MgCl}_{2}, 26 \mathrm{mmol} / \mathrm{L} \mathrm{NaHCO}{ }_{3}, 1.25 \mathrm{mmol} / \mathrm{L} \mathrm{NaH}_{2} \mathrm{PO}_{4}, 10$ $\mathrm{mmol} / \mathrm{L}$ glucose, $2 \mathrm{mmol} / \mathrm{L} \mathrm{CaCl}_{2}$, and $400 \mu \mathrm{mol} / \mathrm{L} \mathrm{L}$-ascorbic acid, with osmolarity at 300-305 mOsm, equilibrated with $95 \% \mathrm{O}_{2} / 5 \% \mathrm{CO}_{2}$. Slices were kept at room temperature (RT) in aCSF equilibrated with $95 \%$ $\mathrm{O}_{2} / 5 \% \mathrm{CO}_{2}$, pH 7.4, until transferred to the microscopy chamber.

Vessel cannulation. PAs were visualized using a $60 \times$ Nikon objective (NIR Apo, $60 \times / 1.0 \mathrm{w}$, DIC N2, $\infty / 0 \mathrm{WD} 2.8$ ) equipped with infrared differential interference contrast (IR-DIC) optics. Cannulas (inner diameter, $1.17 \mathrm{~mm}$; outer diameter, $1.50 \mathrm{~mm}$; G150TF-3, Warner Instruments) were pulled with a micropipette puller (P-97 puller, Sutter Instruments) and mounted onto a micromanipulator. Luminal flow ( $Q)$ was controlled using a syringe pump (PHD 2000, Harvard Apparatus). A pressure transducer was placed just before the cannula for constant pressure monitoring (servo pump; PS/200, Living System Instrumentation), as previously described (Kim and Filosa, 2012). The internal cannula solution consisted of the following: $3 \mathrm{mmol} / \mathrm{L} \mathrm{KCl}, 135 \mathrm{mmol} / \mathrm{L} \mathrm{NaCl}, 1$ $\mathrm{mmol} / \mathrm{L} \mathrm{MgCl}_{2}, 10 \mathrm{mmol} / \mathrm{L}$ glucose, $10 \mathrm{mmol} / \mathrm{L}$ HEPES, $2 \mathrm{mmol} / \mathrm{L}$ $\mathrm{CaCl}_{2}$, and $1 \%$ albumin (Duling et al., 1981) with osmolarity at 300-305 mOsm and $\mathrm{pH} 7.4$ adjusted with $\mathrm{NaOH}$. The tip of the cannula was maneuvered toward the entrance of the arteriole and slowly introduced into the vessel lumen (Kim and Filosa, 2012). Following cannula insertion, $Q$ was increased to $\sim 0.1 \mu \mathrm{l} / \mathrm{min}$ to induce myogenic tone. At the end of the experiment, maximum diameter was obtained by perfusing slices with zero $\mathrm{Ca}^{2+}$ aCSF containing papaverine (100 $\left.\mu \mathrm{mol} / \mathrm{L}\right)$. Diameters are expressed as percentage from maximum.

Astrocytic syncytium loading. Cortical astrocytes (within cortical layers II-IV) in close proximity $(<150 \mu \mathrm{m})$ to a PA were identified with a $60 \times$ water-immersion objective. Patch pipettes were made from thin-walled borosilicate glass (outer diameter, $1.5 \mathrm{~mm}$; internal diameter, $0.86 \mathrm{~mm}$; BF150-86-7.5, Sutter Instrument) and pulled (P-97 puller, Sutter Instru- ment) to resistances between 6 and $8 \mathrm{M} \Omega$. The internal solution for BAPTA loading into astrocytes consisted of the following (in $\mathrm{mmol} / \mathrm{L}$ ): $130 \mathrm{~K}^{+}$gluconate, $10 \mathrm{HEPES}, 10 \mathrm{BAPTA}, 10 \mathrm{KCl}, 0.9 \mathrm{MgCl}_{2}, 4 \mathrm{Mg}_{2} \mathrm{ATP}$, $0.3 \mathrm{Na}_{2} \mathrm{GTP}, 20$ phosphocreatine (see Fig. $3 A, C, E, F$ ). The internal solution for high $\mathrm{Ca}^{2+}$ loading into astrocytes consisted of the following (in $\mathrm{mmol} / \mathrm{L}$ ): $130 \mathrm{~K}^{+}$gluconate, 10 HEPES, 0.2 EGTA, $10 \mathrm{KCl}, 0.9$ $\mathrm{MgCl}_{2}, 0.126 \mathrm{CaCl}_{2}, 4 \mathrm{Mg}_{2} \mathrm{ATP}, 0.3 \mathrm{Na}_{2} \mathrm{GTP}, 20$ phosphocreatine (see Fig. $3 D-F)$. The internal solution for high ATP loading into astrocytes consisted of the following (in mmol/L): $94 \mathrm{~K}^{+}$gluconate, 10 HEPES, 0.2 EGTA, $10 \mathrm{KCl}, 0.9 \mathrm{MgCl}_{2}, 40 \mathrm{Mg}_{2} \mathrm{ATP}, 0.3 \mathrm{Na}_{2} \mathrm{GTP}$ 0.3, 20 phosphocreatine (see Fig. $8 E-G$ ). For all internal solutions, the osmolarity was $291-$ $295 \mathrm{mOsm}$ and $\mathrm{pH}$ adjusted to 7.2 with $\mathrm{KOH}$. To visualize loading of the astrocytic syncytium, $100 \mu \mathrm{mol} / \mathrm{L}$ Alexa 488 was added to the internal solution. Two astrocytes were patched per slice.

Calcium imaging. Experiments were conducted using the Andor Revolution system (Andor Technology) attached to a Nikon microscope (Eclipse FN 1, Nikon) equipped with a laser confocal spinning unit (CSU-X1, Yokogawa) attached to a Sutter filter wheel and an ultrasensitive electron-multiplying CCD camera (iXon ${ }^{\mathrm{EM}}$, Andor Technology; Kim and Filosa, 2012). The microscope chamber was continuously perfused with aCSF using a peristaltic pump (Miniplus 3, Gilson) at a rate of $2 \sim 3 \mathrm{ml} / \mathrm{min}$. Chamber temperature was maintained at $36 \pm 1^{\circ} \mathrm{C}$ using a single line solution heater ( $\mathrm{SH}-28 \mathrm{~B}$, Warner Instruments) connected to a DC power supply (1735A, BK Precision). Cortical slices were incubated at RT in aCSF containing $10 \mu \mathrm{mol} / \mathrm{L}$ Fluo- $4 \mathrm{AM}$ and pluronic acid (1.5 $\mu \mathrm{g} / \mathrm{ml})$. Following a $2 \mathrm{~h}$ incubation period, slices were placed in RT aCSF until needed. Fluorescence images were obtained using a krypton/argon laser at $488 \mathrm{~nm}$ and emitted light at $>495 \mathrm{~nm}$. Images were acquired at $2-4$ frames/s for $\sim 6 \mathrm{~min}$. Acquisition was performed using either $20 \times$, $40 \times$, or $60 \times$ Nikon objectives. For in vitro studies, a subpopulation of astrocytes displaying spontaneous $\mathrm{Ca}^{2+}$ oscillations with high frequencies $(\sim 0.5 \mathrm{~Hz})$ were not included in the analysis, given that such degree of activity was not observed in vivo (in this study and others; Takano et al., 2007; Thrane et al., 2012) and was previously reported in disease conditions (Takano et al., 2007), which led us to conclude that they likely represent an artifact of the slice preparation.

Immunohistochemistry. Male Wistar rats (age P28) were anesthetized and perfused transcardially with $150 \mathrm{ml}$ of $0.01 \mathrm{~mol} / \mathrm{L}$ PBS, $\mathrm{pH}$ 7.3-7.4, and $350 \mathrm{ml}$ of $4 \%$ paraformaldehyde (PFD). Following fixation for 3-4 h in $4 \% \mathrm{PFD}$ at $4^{\circ} \mathrm{C}$, brains were cryoprotected in $0.01 \mathrm{~mol} / \mathrm{L}$ PBS with $30 \%$ sucrose for $72 \mathrm{~h}$ and then frozen at $-80^{\circ} \mathrm{C}$. Using the Leica CM3050 S cryostat (Leica Microsystems), $30 \mu \mathrm{m}$ coronal cortical sections were cut and stored in cryoprotectant solution $(50 \mathrm{mmol} / \mathrm{L}$ PBS, 30\% ethylene glycol, 20\% glycerol) until needed.

Fixed brain slices were blocked for $1 \mathrm{~h}$ in $0.01 \mathrm{~mol} / \mathrm{L}$ PBS containing $0.1 \%$ Triton X-100, $0.04 \% \mathrm{NaN}_{3}$, and $10 \%$ horse serum (Vector Laboratories). Slices were then incubated for $48 \mathrm{~h}$ at RT in a primary antibody mixture containing rabbit anti-TRPV4 (1:200; Lifespan Biosciences), goat anti-AQP4 (1:500; Santa Cruz Biotechnology), and either mouse anti-GFAP (1:5000; Millipore Bioscience Research Reagents) or mouse anti-RECA-1 (1:1000; Serotec). Following primary antibody treatment and three washes with $0.01 \mathrm{~mol} / \mathrm{L}$ PBS, slices were incubated for $4 \mathrm{~h}$ in a secondary antibody mixture containing donkey anti-rabbit CY3 (1:250), donkey anti-goat Alexa Fluor 647 (1:50), and donkey anti-mouse FITC (1:250; secondary antibodies purchased from Jackson ImmunoResearch). Both primary and secondary antibodies were diluted in 0.01 mol/L PBS containing $0.1 \%$ Triton X-100 and $0.04 \% \mathrm{NaN}_{3}$. Slices were mounted using Vectashield (Vector Laboratories). Z-stack images were acquired at an interval of $0.1-0.5 \mu \mathrm{m}$ with a Zeiss LSM 510 confocal scanning microscope using a $63 \times$ oil-immersion objective and the Zeiss LSM510 program (Carl Zeiss Microscopy).

Preparation of mice for in vivo imaging. Craniotomy for the optical window follows standard protocols approved by the animal care and use committee of Georgia Regents University. Mice were anesthetized with an intraperitoneal injection of urethane $(1.5 \mathrm{mg} / \mathrm{g}$ body weight). Heart rate (HR, 450-650 beats/min) and oxygen saturation level (>90\%) were monitored using a MouseOx pulse oximeter (Starr Life Sciences) and body temperature was maintained at $37^{\circ} \mathrm{C}$ with a heating pad (Sunbeam). Depth of anesthesia as assessed by toe pinch and HR monitoring 
was maintained with $10 \%$ of the initial urethane dose if necessary. To visualize vessels, a $0.1 \mathrm{ml}$ bolus of $5 \%$ (w/v) Texas Red dextran (70 kDA; Invitrogen) in $0.9 \% \mathrm{NaCl}$ was injected into the tail vein. In some experiments, arteriole visualization was conducted through topical application of Alexa 633 as previously reported (Shen et al., 2012). The skin covering the cranium above the somatosensory cortex was removed and a custom-made $1.3-\mathrm{cm}$-diameter plastic ring was glued with dental acrylic cement (Co-Oral-Ite Dental) to stabilize the head during craniotomy and imaging with a headholder attached to a baseplate. A dental drill (Midwest Stylus Mini 540S, Dentsply International) was used to thin the circumference of a 2-4-mm-diameter circular region of the skull over somatosensory cortex centered at stereotaxic coordinates $-1.8 \mathrm{~mm}$ from bregma and $2.8 \mathrm{~mm}$ lateral. The thinned bone was then lifted with forceps. Fluo-4 AM ( $1 \mathrm{mmol} / \mathrm{L})$, pluronic acid $(20 \%)$, and, in some instances, Alexa $633(200 \mu \mathrm{mol} / \mathrm{L})$ were mixed with $80 \mu \mathrm{l}$ of cortex buffer containing (in mmol/L) the following: $135 \mathrm{NaCl}, 5.4 \mathrm{KCl}, 1 \mathrm{MgCl}_{2}, 1.8$ $\mathrm{CaCl}_{2}$, and $5 \mathrm{HEPES}, \mathrm{pH}$ 7.3. This was applied to the cortical surface for $1 \mathrm{~h}$ to allow loading. The solution was then removed, the surface of the brain was washed with cortex buffer, and an optical chamber was constructed by covering the brain with a thin layer of $1.5 \%$ agarose prepared in cortex buffer. The chamber was sealed with a circular glass coverslip (\#1943-00005, Bellco). The baseplate containing the headholder with the mouse resting on a heating pad was affixed to the Luigs and Neumann microscope stage for imaging.

Two-photon laser scanning microscopy. Two-photon laser scanning microscopy images were collected with infrared-optimized $40 \times / 0.8 \mathrm{nu}$ merical aperture water-immersion objective (Carl Zeiss) using the LSM 510 NLO META multiphoton system (Zeiss) mounted on the motorized upright Axioscope-2FS microscope (Zeiss). The scan module is directly coupled with the Spectra-Physics Ti:sapphire broadband mode-locked laser (Mai-Tai) tuned to $825 \mathrm{~nm}$ for two-photon excitation. The majority of astrocytes used for imaging were from cortical layers I/II. Due to the possibility of movement during blood pressure changes with phenylephrine (Phe), three-dimensional time-lapse high-magnification images were taken at 2-3 $\mu \mathrm{m}$ increments (with $\sim 13$ sections per $z$-stack) using $3 \times$ optical zoom, yielding a nominal spatial resolution of 6.86 pixels $/ \mu \mathrm{m}$ (12 bits/pixel, $0.91 \mu$ s pixel time) across a $75 \times 75 \mu \mathrm{m}$ imaging field. Low-magnification images were taken with $0.7 \times$ optical zoom, resulting in a nominal spatial resolution of 3.19 pixels/ $\mu \mathrm{m}$ ( 8 bits/pixel, $2.51 \mu \mathrm{s}$ pixel time) across a $318 \times 318 \mu \mathrm{m}$ imaging field. Emitted light was detected by internal photomultiplier tubes of the scan module with the pinhole entirely opened. If shifting of the focal plane occurred, the field of focus was adjusted and recentered before acquiring image stacks.

Drugs and chemicals. All drugs, with the exception of Fluo4 AM, Alexa 488, (Invitrogen), HC067047, paxilline (Tocris Bioscience), 4- $\alpha$-PDD (LC Laboratories), bovine serum albumin (Fisher Scientific), N-hydroxy-N' -(4butyl-2-methylphenyl)-formamidine (HET0016), and suramin (Cayman Chemical Company), were purchased from Sigma-Aldrich. Drugs were bath applied, unless otherwise specified. The selected dosages and concentrations were based on extensive published literature.

Data analysis. Arteriolar diameter data (IR-DIC) and in vivo studies were analyzed using Sparkan (Adrian Bonev, University of Vermont). For cannulation experiments, tone was calculated as the percentage constriction from the maximal diameter induced by zero $\mathrm{Ca}^{2+}$ aCSF and papaverine $(100 \mu \mathrm{mol} / \mathrm{L})$. Analysis for electrophysiological recordings was performed using Clampfit 10.2, pClamp version 10.2 (Axon Instruments). $\mathrm{Ca}^{2+}$ imaging was analyzed using Sparkan software. Fractional fluorescence $\left(F / F_{\mathrm{o}}\right)$ was determined by dividing the fluorescence intensity $(F)$ within a region of interest (ROI) by a baseline fluorescence value $\left(F_{\mathrm{o}}\right)$ determined from $\sim 20$ images (for in vitro studies) or $\sim 5$ images (from in vivo studies) showing no activity. The area under the curve (AUC), for a basal period and during the $\mathrm{Ca}^{2+}$ response, was determined as the integral over time within an ROI ( $10 \times 10$ pixels $)$ on a cell exhibiting $\mathrm{Ca}^{2+}$ activity. Prism software 5 (Graphpad) was used for all statistical analyses. All values are expressed as mean \pm SEM. Experiments were conducted $\geq 4 \times$ in $\geq 3$ different rats/mice. Differences between two means within groups were determined using Student's paired $t$ test. Between-group differences (e.g., TRPV4 ${ }^{+/+}$vs TRPV4 ${ }^{-/-}$) were compared using unpaired $t$ tests. Statistical significance was tested at $95 \%$ $(p<0.05)$ confidence level and is denoted with asterisks in figures: ${ }^{\star} p<$ $0.05,{ }^{* *} p<0.01$, or ${ }^{* * *} p<0.001$. For in vivo experiments, the technician was blinded to the group type.

\section{Results}

\section{Flow/pressure-induced PA constriction is rapidly sensed by perivascular astrocytes}

Given the intimate anatomical association between blood vessels and astrocytes (Kacem et al., 1998; Simard et al., 2003), we first tested whether astrocyte activity (changes in intracellular $\mathrm{Ca}^{2+}$ ) is altered by flow/pressure-evoked changes in PA vascular tone (Toth et al., 2011; Kim and Filosa, 2012). PAs were perfused and pressurized (Kim and Filosa, 2012) until myogenic tone developed in rat cortical slices loaded with the $\mathrm{Ca}^{2+}$ indicator dye Fluo-4 AM (100 $\mu \mathrm{mol} / \mathrm{L})$. We found that mild (from 0.1 to 0.3 $\mu \mathrm{l} / \mathrm{min}$; Fig. $1 A-D$ ) and high (from 0.1 to $0.5-0.8 \mu \mathrm{l} / \mathrm{min}$; Fig. $1 E--H)$ increases in flow $(Q)$ for short ( $\leq 60 \mathrm{~s}$ ) and longer (120 s) stimulation periods evoked increases in astrocytic $\mathrm{Ca}^{2+}$. For mild increases in $Q(\leq 60 \mathrm{~s})$, both peak amplitude $(\Delta 0.083 \pm 0.028$ $F / F_{0}, p=0.0052, n=53$; Fig. $1 A, B$, left $)$ and AUC $(\Delta 1.62 \pm 0.47$ $F / F_{0} \cdot s, p=0.0010, n=53$; Fig. $1 A, C$, left) were significantly increased. The averaged response onset was $18.99 \pm 2.32 \mathrm{~s}(n=$ 53 ) following the change in $Q$ (Fig. $1 D$, left). Increasing the stimulus duration (120 s) also evoked a significant increase in peak amplitude $\left(\Delta 0.074 \pm 0.021 F / F_{0}, p=0.0007, n=66\right.$; Fig. $1 B$, right $)$ and $\mathrm{AUC}\left(\Delta 3.38 \pm 0.40 \mathrm{~F} / \mathrm{F}_{0} \cdot \mathrm{s}, p<0.0001, n=66\right.$; Fig. $1 C$, right). The averaged response onset was $15.13 \pm 1.98 \mathrm{~s}(n=$ 66 ) following the change in $Q$ (Fig. $1 D$, right). Likewise, a significant increase in peak amplitude $\left(\Delta 0.119 \pm 0.013 F / F_{0}, p<\right.$ $0.0001, n=104$; Fig. $1 E, F$, left $)$ and $\operatorname{AUC}\left(\Delta 1.85 \pm 0.21 \mathrm{~F} / F_{0} \cdot \mathrm{s}\right.$, $p<0.0001, n=104$; Fig. $1 E, G$, left) were observed in response to high $Q(\uparrow \uparrow \uparrow Q)$ increases ( $\leq 60 \mathrm{~s})$. The average response onset was $18.90 \pm 1.51 \mathrm{~s}(n=104)$ following the change in $Q$ (Fig. $1 H$, left). Increasing the stimulus duration (120 s) also evoked a significant increase in both peak amplitude $\left(\Delta 0.137 \pm 0.040 \mathrm{~F} / \mathrm{F}_{0}\right.$, $p=0.0053, n=13$; Fig. $1 F$, right $)$ and $\operatorname{AUC}\left(\Delta 4.81 \pm 0.78 \mathrm{~F} / F_{0}\right.$. s, $p<0.0001, n=13$; Fig. $1 G$, right). The average response onset was $17.98 \pm 4.69 \mathrm{~s}(n=13)$ following the change in $Q$ (Fig. $1 H$, right). The stimulus duration ( $\leq 60 \mathrm{~s}$ vs $120 \mathrm{~s}$ ) did not have an effect on the peak amplitude of the $\Delta F / F_{0}$ between mild ( $\uparrow Q$ ) and high $(\uparrow \uparrow \uparrow Q$ ) increases $(p=0.8$ vs $p=0.66$ unpaired $t$ test, respectively). On the other hand, the stimulus duration ( $\leq 60 \mathrm{~s}$ vs $120 \mathrm{~s}$ ) resulted in significant differences with the $\Delta \mathrm{AUC}$ $(\Delta 1.84 \pm 0.62$ for $\uparrow Q, p<0.0036$ and $\Delta 2.96 \pm 0.65$ for $\uparrow \uparrow \uparrow Q, p<0.0001$, unpaired $t$ test) and the duration of the response $(\Delta 148.36 \pm 7.62$ for $\uparrow Q, p<0.0001$ and $\Delta 151.52 \pm$ 8.32 for $\uparrow \uparrow \uparrow Q, p<0.0001$, unpaired $t$ test), suggesting that the longer the stimulus period, the greater and the longer the astrocyte $\mathrm{Ca}^{2+}$ response.

To gain better insight into the time course of cellular responses during the hemodynamic stimulus, we monitored $\mathrm{Ca}^{2+}$ activity in VSMCs alone and, when possible, simultaneously with that of astrocytes. Mild increases in $Q$ induced a significant increase in VSMC Ca ${ }^{2+}$ oscillation frequency $(\Delta 0.25 \pm 0.02 \mathrm{~Hz}$, $n=44, p<0.0001)$ and $F / F_{0}(\Delta 0.11 \pm 0.02, n=44, p<0.0001$; Fig. $2 A, B)$, consistent with flow/pressure-evoked constriction (Toth et al., 2011; Kim and Filosa, 2012). Consistently, AUC increased by $\Delta 0.55 \pm 0.06 \mathrm{~F} / \mathrm{F}_{0} \cdot \mathrm{s}(p<0.0001, n=44$; Fig. $2 C)$. Responses occurred $4.86 \pm 0.48 \mathrm{~s}(n=44)$ after the change in $Q$ (Fig. 2D). In exceptional cases where VSMCs and astrocytes were in the same focal field, simultaneous $\mathrm{Ca}^{2+}$ measurements from these cells were obtained in response to increases in $Q$. Consistent with flow/pressure-induced PA constriction (Bagi et al., 2001; 
Bryan et al., 2001; Kim and Filosa, 2012), an increase in $Q$ rapidly (3.52 $\pm 0.91 \mathrm{~s})$ increased VSMC $\mathrm{Ca}^{2+}$ oscillation frequency, preceding changes in astrocytic $\mathrm{Ca}^{2+}$ by $15.84 \pm 6.40 \mathrm{~s}(n=3$ experiments; Fig. 2E,F). These data support the activation of astrocytes by flow/pressureevoked vascular responses.

Astrocytes contribute to flow/pressureinduced PA tone adjustments

To better understand the physiological significance for hemodynamic stimuliinduced astrocyte activation, we investigated whether the flow/pressure stimulus prompted activated astrocytes to release vasoactive signals, thus contributing to the modulation of PA myogenic responses. We measured vascular responses in perfused/pressurized PAs after first loading the astrocytic syncytium with the $\mathrm{Ca}^{2+}$ chelator BAPTA $(10 \mathrm{mmol} / \mathrm{L})$ and Alexa $488(100 \mu \mathrm{mol} / \mathrm{L})$ via a patch pipette (Fig. $3 A$ ). To ensure repeatability of vascular responses, we first measured PA diameter changes to two successive increases in flow/pressure (Fig. 3B). Baseline vascular tone between stimuli was not changed $(23.54 \pm 2.90 \%$ vs $24.19 \pm$ $2.42 \%, p=0.56$, paired $t$ test, $n=6$; Fig. $3 E)$. Flow/pressure-evoked vascular responses were measured at both the time of peak constriction (typically within $3 \mathrm{~min}$ after the increase in flow) and at minute 10 , the last time point before flow rate was brought back to control levels. No differences were observed for the initial flow/ pressure-evoked constriction between the first and second stimulus $(\Delta 20.83 \pm$ $2.92 \%$ vs $\Delta 19.92 \pm 3.18 \%, p=0.31$, paired $t$ test, $n=6$ ) or for the sustained tone measured at the end of the stimulus protocol $(\Delta 20.89 \pm 3.33 \%$ vs $\Delta 20.65 \pm$ $3.46 \%, p=0.79$, paired $t$ test, $n=6$; Fig. $3 F)$, which is consistent with the ability of $\mathrm{PA}$ to respond similarly to two consecutive stimuli. However, within $20 \mathrm{~min}$ of BAPTA loading (assessed by an increased number of Alexa 488-loaded astrocytes), baseline vascular tone was significantly reduced by $\Delta 12.48 \pm 3.56 \%(p<0.05, n=$ 6; Fig. $3 C, E)$. Although the initial increase in flow/pressure-evoked PA tone was similar before and after BAPTA loading $(\Delta 20.01 \pm 3.79 \%$ vs $\Delta 20.86 \pm 4.46 \%, p>$ $0.05, n=6)$, the ability of PAs to sustain tone throughout the duration of the hemodynamic challenge was blunted in BAPTAloaded slices $(\Delta 20.65 \pm 3.46 \%$ vs $\Delta 8.14 \pm 2.94 \%, p=0.0357$, unpaired $t$ test, $n=6$; Fig. $3 C, F)$. These results support a role for astrocytes in the sustained (vs acute) phase of hemodynamicinduced adjustments in vascular tone.

As previously demonstrated, an increase in astrocytic $\mathrm{Ca}^{2+}$ constitutes an important step toward astrocytic regulation of vas-
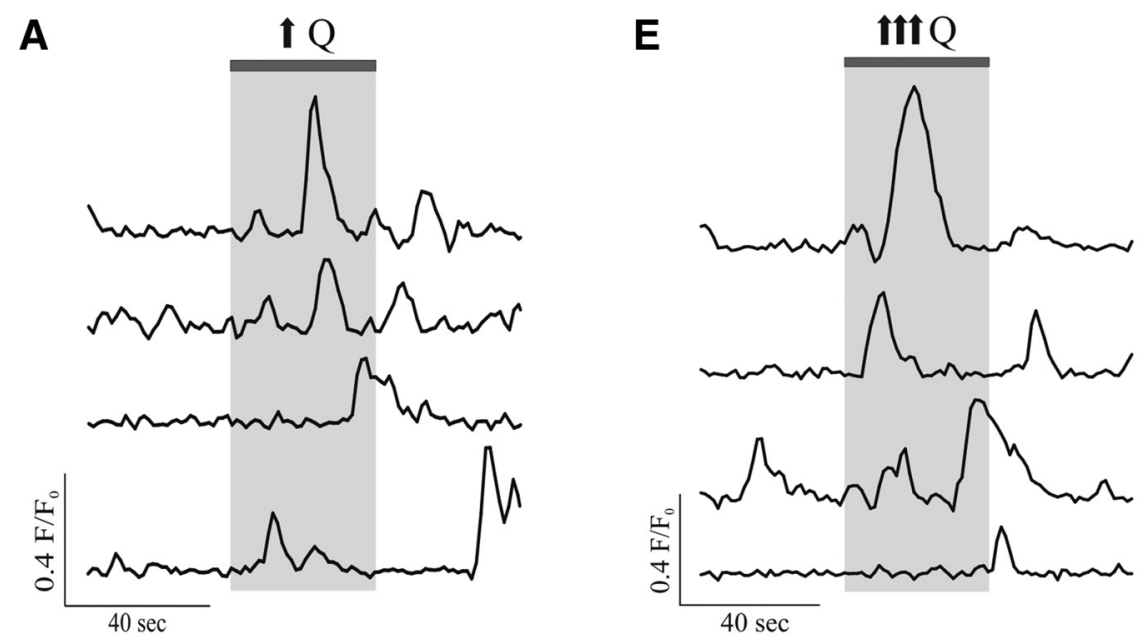

Baseline $\square$ Stimulus response

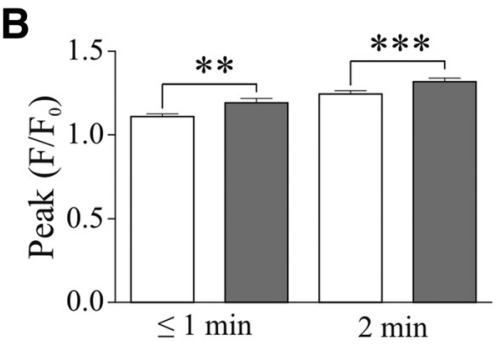

$\mathbf{F}$
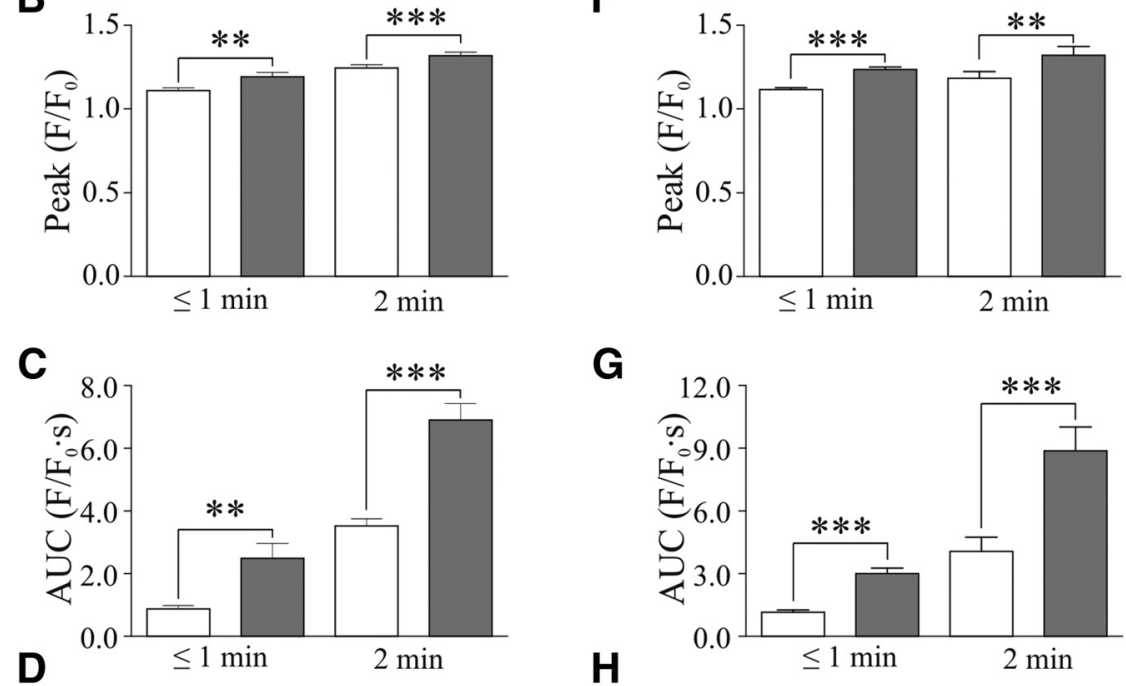

G
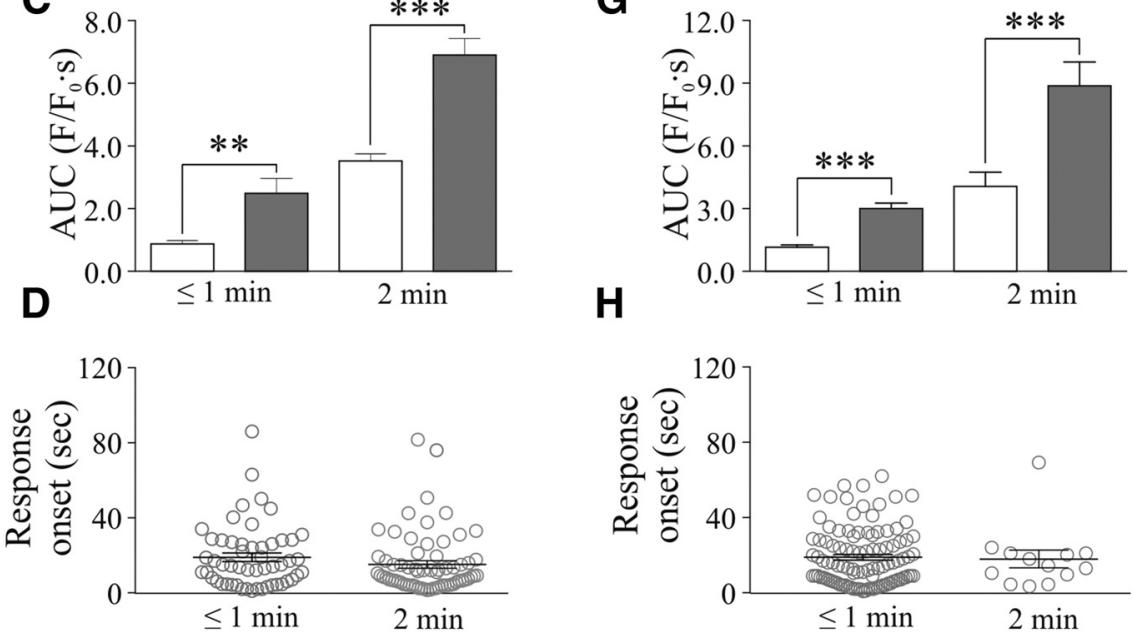

Figure 1. Flow/pressure-evoked increases in astrocytic $\mathrm{Ca}^{2+}$ activity. $A$, Representative traces of astrocytic $\mathrm{Ca}^{2+}$ activity in response to a mild increase in $Q(\uparrow Q) . B$, Averaged peak amplitude (peak $F / F_{0}$ ) during baseline (open bar) and stimulus response period (gray bar) for short ( $\leq 1 \mathrm{~min}$, left) or a $2 \mathrm{~min}$ (right) stimulus. C, Averaged AUC during baseline (open bar) and stimulus response period (gray bar) for short ( $\leq 1 \mathrm{~min}$, left) or a $2 \mathrm{~min}$ (right) stimulus. $\boldsymbol{D}$, Onset time for astrocytic $\mathrm{Ca}^{2+}$ responses. $\boldsymbol{E}$, Representative traces of astrocytic $\mathrm{Ca}^{2+}$ activity in response to a high increase in $Q(\uparrow \uparrow \uparrow Q)$. $\boldsymbol{F}$, Averaged peak amplitude (peak $F / F_{0}$ ) during baseline (open bar) and stimulus response period (gray bar) for short ( $\leq 1 \mathrm{~min}$, left) or a $2 \mathrm{~min}$ (right) stimulus. $G$, Averaged AUC during baseline (open bar) and stimulus response period (gray bar) for short ( $\leq 1 \mathrm{~min}$, left) or a $2 \mathrm{~min}$ (right) stimulus. $\boldsymbol{H}$, Onset time for astrocytic $\mathrm{Ca}^{2+}$ responses. ${ }^{* *} p<0.01,{ }^{* * *} p<0.001$.

cular tone. Thus we measured flow/pressure-evoked vascular responses when the astrocytic syncytium was loaded with a high $\mathrm{Ca}^{2+}$ concentration $(126 \mu \mathrm{mol} / \mathrm{L})$ in the patch pipette (Fig. $3 D$ $F$ ). Following an equilibration period to allow loading (assessed by an increased number of Alexa 488-loaded astrocytes), flow/ pressure-evoked vascular responses were assessed. Baseline vascular tone was not changed $(\Delta 22.92 \pm 4.55 \%$ vs $\Delta 23.14 \pm 4.55 \%$, $p=0.6308, n=4$; Fig. $3 E)$. However, the percentage change in 


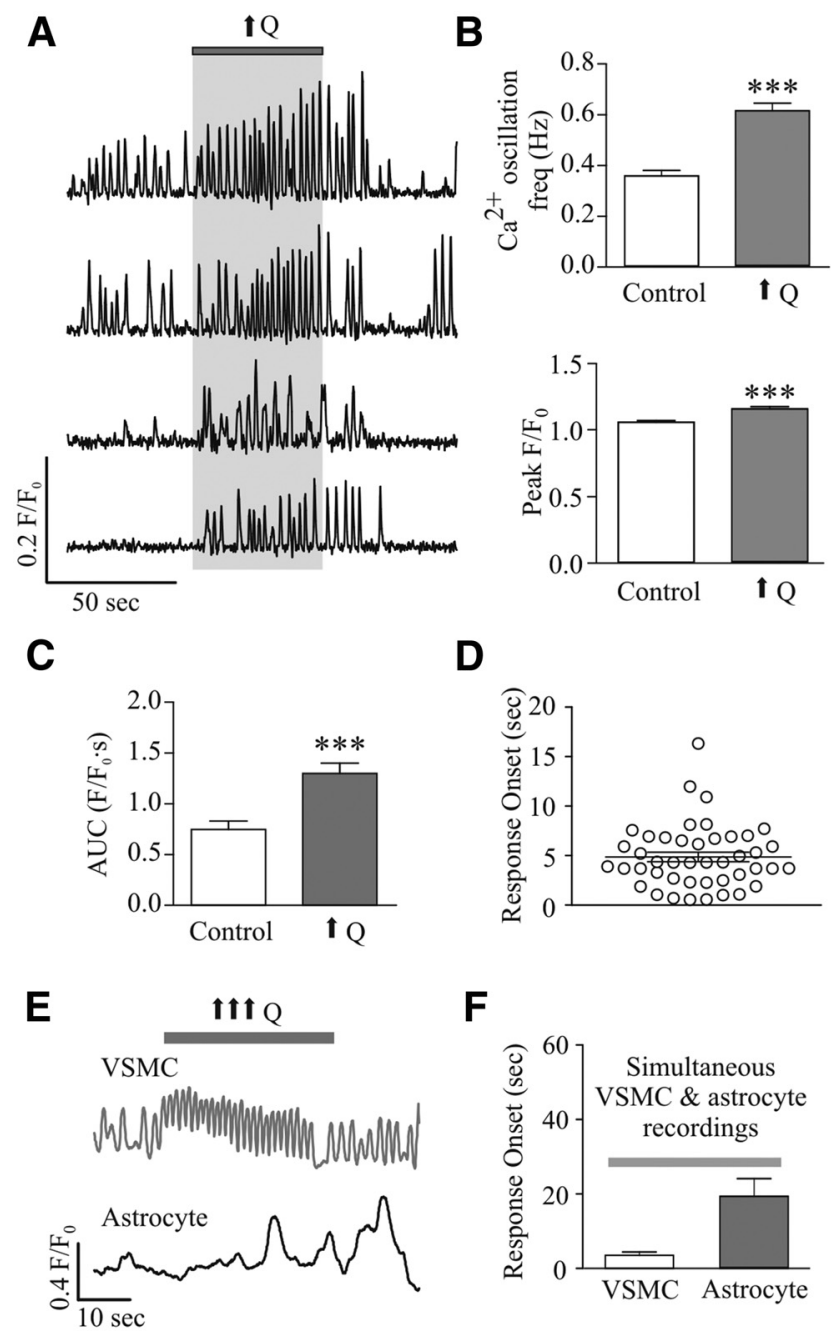

Figure 2. Flow/pressure-evoked increases in VSMC $\mathrm{Ca}^{2+} . A$, Representative trace of VSMC $\mathrm{Ca}^{2+}$ activity in response to increases in flow $(\uparrow Q) . B$, Averaged VSMC Ca ${ }^{2+}$ oscillation frequency (top) and peak $F / F_{0}$ amplitude (bottom) in response to $\uparrow Q$. C, Averaged AUC during baseline (open bar) and stimulus response period (gray bar) for VSMC $\mathrm{Ca}^{2+}$ responses. $\boldsymbol{D}$, Onset time for VSMC $\mathrm{Ca}^{2+}$ responses to $\uparrow Q$.E, Representative traces showing simultaneous recordings of $\mathrm{Ca}^{2+}$ activity in a VSMC and an astrocyte in response to $\uparrow \uparrow \uparrow Q . \boldsymbol{F}$, Averaged response onset times for simultaneous VSMC and astrocyte $\mathrm{Ca}^{2+}$ activity. ${ }^{* *} p<0.001$.

tone ( $\Delta$ tone) at the time of peak constriction (within 3 min from stimulus onset) was significantly higher when compared with controls $(\Delta 19.92 \pm 3.18 \%$ vs $\Delta 41.56 \pm 5.16 \%, p=0.0052$, unpaired $t$ test). The magnitude of the flow/pressure-evoked constriction was not sustained, however, throughout the duration of the stimulus $(\Delta 23.80 \pm 3.97 \%$ at minute 8 from stimulus onset vs $\Delta 41.56 \pm 5.16 \%$ at minute 3 from stimulus onset, $p=0.0070$, paired $t$ test; Fig. $3 D-F$ ). These data support astrocyte $\mathrm{Ca}^{2+}$ signaling for the regulation of basal vascular tone and as active modulators of flow/pressure-evoked PA constrictions.

\section{Astrocytic TRPV4 channels contribute to PA tone adjustments}

Mechanosensitive TRPV4 channels have been previously reported to be expressed in astrocytes (Benfenati et al., 2007), cerebral vessels (Earley et al., 2005; Marrelli et al., 2007), and the peripheral vasculature (Köhler et al., 2006; Earley et al., 2009). Thus, we used a combination of complementary approaches to elucidate their relative contribution to flow/pressure-evoked increases in astrocyte $\mathrm{Ca}^{2+}$. In pial arterioles, TRPV4 channel ex- pression was found primarily on the luminal side of the vessels, where it colocalized with the EC marker RECA-1 (Fig. 4A, left column). On the other hand, TRPV4 channel staining was limited to the adventitial side of PAs, where it colocalized with the astrocyte process marker GFAP and the astrocyte endfoot marker AQP4, in agreement with previous studies (Benfenati et al., 2007, 2011; Fig. 4A, middle column). As shown in Figure $4 A$ (right column), a PA cross-sectional view further confirmed TRPV4 channel expression on the abluminal side of the vessels as opposed to the endothelium. To corroborate our immunofluorescence data showing TRPV4 channel expression in cortical perivascular astrocytes, we next evaluated channel function using the selective TRPV 4 channel agonist $4 \alpha \mathrm{PDD}(5 \mu \mathrm{mol} /$ L). Bath-applied $4 \alpha \mathrm{PDD}$ evoked significant increases in astrocytic $\mathrm{Ca}^{2+}$ oscillation frequency $(\Delta 0.01 \pm 0.003 \mathrm{~Hz}, p<$ $0.0001, n=38)$ and amplitude $\left(\Delta 0.37 \pm 0.05 F / F_{0}, p<0.0001\right.$, $n=38$; Fig. $4 B, C$ ).

To test for TRPV4 channel contribution to hemodynamic adjustments in myogenic tone, we then measured flow/pressureevoked vascular responses in the absence and then presence of the TRPV4 channel blocker HC067047 (5 $\mu \mathrm{mol} / \mathrm{L} ; 20$ min perfusion). Flow/pressure-evoked vascular responses were comparable to those observed in BAPTA experiments (Fig. 3). HC067047 treatment had no effect on the resting tone of PAs $(\Delta 4.81 \pm$ $1.70 \%, p>0.05, n=6$; Fig. $5 A-C$ ). However, while flow/ pressure-evoked vasoconstriction was sustained throughout the duration of the hemodynamic stimulus in the absence of HC067047 ( $\Delta 23.59 \pm 3.44 \%$ at peak constriction vs $\Delta 22.14 \pm$ $2.61 \%$ at minute 8 of the hemodynamic stimulus, $n=6$; Fig. $5 D$, left), the sustained component was significantly reduced in the presence of $\mathrm{HC} 067047(\Delta 22.37 \pm 3.84 \%$ at peak constriction vs $\Delta 9.88 \pm 1.99 \%$ at minute 8 of the stimulus, $p<0.001, n=6$; Fig. $5 D$, right).

We then assessed flow/pressure-evoked vasconstriction following two incremental increases in flow [from 0.1 to $0.5 \mu \mathrm{l} / \mathrm{min}$ $(\uparrow Q)$ and from 0.5 to $0.8 \mu \mathrm{l} / \mathrm{min}(\uparrow \uparrow \uparrow Q)]$ in PA from TRPV4 ${ }^{+/+}$and TRPV $4^{-1-}$ mice (Fig. 6). Similar to observations from rat PA vascular responses (Fig. 5), flow/pressure-evoked vasoconstrictions were sustained throughout the duration of the hemodynamic stimulus in TRPV $4^{+/+}$mice $(\Delta 24.41 \pm 2.03 \%$ for $\uparrow Q$ and $\Delta 28.69 \pm 1.23 \%$ for $\uparrow \uparrow \uparrow Q$ at peak onset constriction vs $\Delta 24.32 \pm 1.70 \%$ for $\uparrow Q$ and $\Delta 28.19 \pm 3.15 \%$ for $\uparrow \uparrow \uparrow Q$ at minute 8 of the stimulus; $p=0.8199$ for $\uparrow Q$ and $p=0.8379$ for $\uparrow \uparrow \uparrow Q, n=4$; Fig. $6 A, B, D)$. On the other hand, in TRPV $4^{-1-}$ mice, the sustained component of the flow/pressure-evoked vasoconstriction was significantly reduced from the onset response for both $\uparrow Q$ and $\uparrow \uparrow \uparrow Q$ stimuli $(\Delta 13.49 \pm 3.19 \%$ for $\uparrow Q$ and $\Delta 15.53 \pm 4.70 \%$ for $\uparrow \uparrow \uparrow Q$ at peak onset constriction vs $\Delta 8.42 \pm 3.30 \%$ for $\uparrow Q$ and $\Delta 8.94 \pm 3.48 \%$ for $\uparrow \uparrow \uparrow Q$ at minute 8 of the stimulus; $p=0.0003$ for $\uparrow Q$ and $p=0.0192$ for $\uparrow \uparrow \uparrow Q, n=5$; Fig. $6 A, B, D)$. Additionally, significant differences were observed between groups (TRPV $4^{+/+}$vs TRPV $4^{-1-}$ ) at peak onset constriction ( $p=0.03$ for $\uparrow Q$ and $p=0.045$ for $\uparrow \uparrow \uparrow Q$ ) and at minute 8 of the stimulus ( $p=0.006$ for $\uparrow Q$ and $p=0.005$ for $\uparrow \uparrow \uparrow Q)$. Baseline vascular tone was not changed between groups $(\Delta 18.44 \pm 0.81 \%$ vs $\Delta 18.14 \pm 3.24 \%$, $p=0.9385$, unpaired $t$ test; Fig. $6 C$ ). These results suggest a TRPV4-dependent astrocytic $\mathrm{Ca}^{2+}$ signaling mechanism for the maintenance of flow/pressure-evoked constrictions.

Consistent with the above findings, we also show that flow/ pressure-evoked vascular responses increased astrocytic $\mathrm{Ca}^{2+}$ activity in TRPV4 ${ }^{+/+}$mice and TRPV4 ${ }^{-1-}$ mice (Fig. $6 E-I$ ). In TRPV4 ${ }^{+/+}$mice, a significant increase was observed in peak $F / F_{0}$ 
A

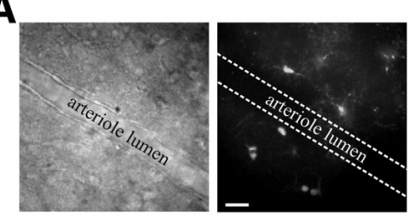

B

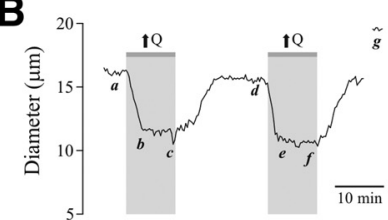

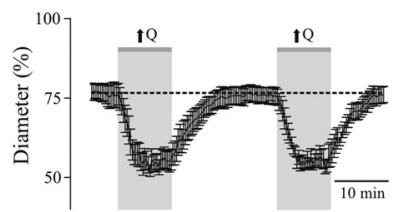

C
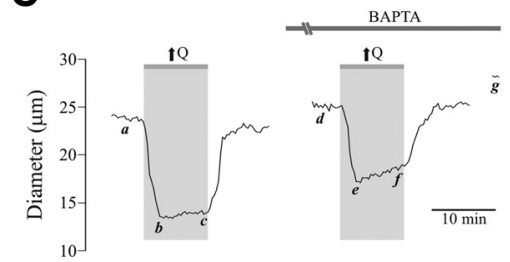

D

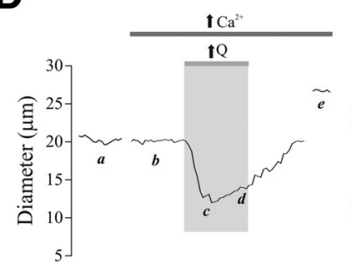

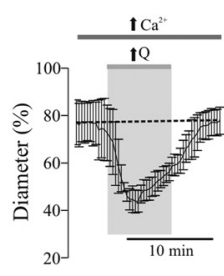

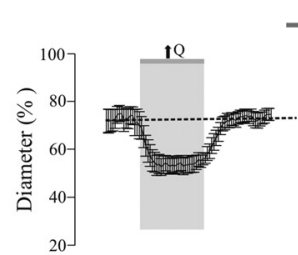

E

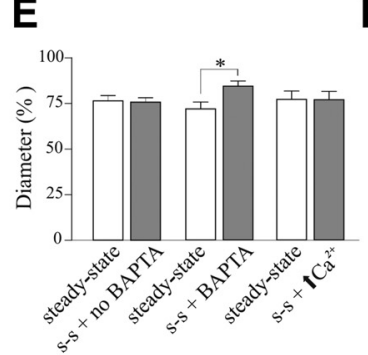

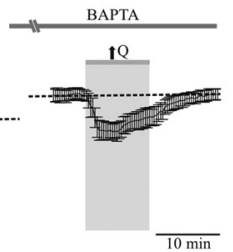

$\mathbf{F}$

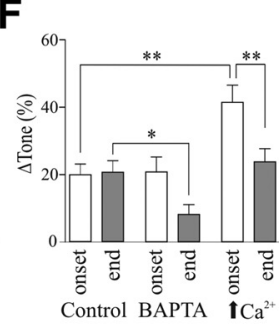

Figure 3. Flow/pressure-evoked increases in PA tone. $\boldsymbol{A}$, Representative DIC image of a perfused PA (left) and BAPTA/Alexa 488-loaded astrocytes (right; scale bar, $25 \mu \mathrm{m}$ ). $\boldsymbol{B}$, Representative trace (left) and averaged (right) diameter change evoked by two successive increases in flow [ $\uparrow Q ; a, d$ : steady-state (s-s) tone; $b$, e: peak constriction at stimulus ( $\uparrow Q)$ onset; $c, f$ : tone at the end of $\uparrow Q$ stimulus; $g$ : maximum diameter in zero $\mathrm{Ca}^{2+}+$ papaverine]. $C$, Representative trace [left, $a, d: s$-s tone; $b, e$ : peak constriction at stimulus ( $\uparrow Q$ ) onset; $c, f$ : tone at the end of $\uparrow Q$ stimulus; $g$ : maximum diameter in zero $\mathrm{Ca}^{2+}+$ papaverine] and averaged diameter (right, percentage from maximum) changes in response to $\uparrow Q$ before and after BAPTA loading of the astrocytic syncytium. $\boldsymbol{D}$, Representative trace (left; $a, b$ : s-s tone; $c$ : peak constriction at stimulus ( $\uparrow Q$ ) onset; $d$ : tone at the end of $\uparrow Q$ stimulus; $e$ : maximum diameter in zero $C^{2+}+$ papaverine) and averaged diameter change (right) in response to $\uparrow Q$, before and after high $\mathrm{Ca}^{2+}$ loading into the astrocytic syncytium. $E$, Averaged $s$-s tone (percentage from maximum) without or with treatment (BAPTA or high $\mathrm{Ca}^{2+}$ in astrocytes). $\boldsymbol{F}$, Averaged $\Delta$ tone (percentage) at the onset and end of the $\uparrow Q$ stimulus. ${ }^{*} p<0.05,{ }^{* *} p<0.001$.

$\left(\Delta 0.110 \pm 0.026 \mathrm{~F} / \mathrm{F}_{0}, p<0.0001, n=71\right.$; Fig. $6 E, F$, left $)$ and $\operatorname{AUC}\left(\Delta 4.66 \pm 0.35 \mathrm{~F} / \mathrm{F}_{0} \cdot \mathrm{s}, p<0.0001, n=71\right.$; Fig. $6 E, \mathrm{G}$, left $)$. Responses occurred $13.84 \pm 1.46 \mathrm{~s}(n=71)$ after the change in $Q$ (Fig. $6 H$, left). In TRPV4 ${ }^{-l-}$ mice, a significant increase was observed in peak $F / F_{0}\left(\Delta 0.097 \pm 0.016 F / F_{0}, p<0.0001, n=13\right.$; Fig. $6 F$, right $)$ and $\operatorname{AUC}\left(\Delta 2.28 \pm 0.40 F / F_{0} \cdot s, p=0.0001, n=13\right.$; Fig. $6 G$, right). Responses occurred $18.92 \pm 7.00 \mathrm{~s}(n=13$; Fig. $6 H$, right) after the change in $Q$. Notably, the baseline AUC between TRPV4 $^{+/+}$and TRPV4 ${ }^{-/-}$mice $\left(2.11 \pm 0.11 \mathrm{~F} / \mathrm{F}_{0} \cdot \mathrm{s}\right.$ vs $1.47 \pm 0.13 \mathrm{~F} / \mathrm{F}_{0} \cdot \mathrm{s}, p=0.0188$, unpaired $t$ test) as well as the flow/pressure-evoked change in the AUC $\left(\Delta 4.66 \pm 0.35 \mathrm{~F} / \mathrm{F}_{0} \cdot \mathrm{s}\right.$ vs $\Delta 2.28 \pm 0.40 \mathrm{~F} / \mathrm{F}_{0} \cdot \mathrm{s}, p=0.0062$, unpaired $t$ test) were significantly reduced in TRPV4 ${ }^{-1-}$ mice (Fig. $6 G$ ). Moreover, a significant reduction in the mean number of astrocytes responding to $\uparrow Q$ was observed in TRPV4 ${ }^{-1-}$ mice compared with TRPV4 ${ }^{+1+}$ mice $(1.30 \pm 0.50$ vs $7.89 \pm 1.23, p<0.0001$, unpaired $t$ test; Fig. $6 I)$.

\section{Astrocyte-derived signals mediate PA tone adjustments}

To address the underlying signaling mechanism by which astrocytes contribute to flow/pressure-evoked adjustments in vascular tone, vascular responses were tested in the presence of blockers against signaling pathways previously associated with astrocytemediated vasoconstriction, namely elevated $\mathrm{K}^{+}$(Girouard et al., 2010), 20-HETE (Mulligan and MacVicar, 2004), and ATP (Kur and Newman, 2014). Twenty minute perfusion with the largeconductance $\mathrm{Ca}^{2+}$-activated $\mathrm{K}^{+}(\mathrm{BK})$ channel blocker paxilline $(2 \mu \mathrm{mol} / \mathrm{L})$ and the $\mathrm{K}_{\mathrm{ir}}$ channel blocker $\mathrm{BaCl}_{2}(100 \mu \mathrm{mol} / \mathrm{L})$ induced a significant increase in baseline tone $(\Delta 23.35 \pm 0.72 \%$, $p<0.001, n=5$; Fig. $7 A-C$ ), thus demonstrating the importance of $\mathrm{BK}$ and/or $\mathrm{K}_{\mathrm{ir}}$ channel function on the regulation of PA resting tone. These blockers, however, had no effect on the magnitude of flow/pressure-induced constrictions at the onset $(\Delta 17.63 \pm$ $2.13 \%$ vs $\Delta 15.61 \pm 1.21 \%, p>0.05, n=5$; Fig. $7 D$ ) or during the sustained phase $(\Delta 16.70 \pm 1.84 \%$ vs $\Delta 15.54 \pm 1.51 \%, p>0.05$, $n=5$, data collected at minute 8 of the stimulus; Fig. $7 D$ ), suggesting a lack of contribution for $\mathrm{K}^{+}$signaling in flow/pressureevoked increases in PA vascular tone.

Previous findings have shown that flow-induced (Toth et al., 2011) and astrocyte-induced (Mulligan and MacVicar, 2004) arteriole constriction following increases in astrocytic intracellular $\mathrm{Ca}^{2+}$ can be mediated by 20-HETE signaling. Thus, we measured flow/pressure-induced vascular responses before and after perfusion of the selective CYP4A inhibitor HET0016 (100 nmol/L; 20 min perfusion; Fig. 7E-H). HET0016 treatment had no effect on the resting tone of PAs $(\Delta 0.41 \pm 1.56 \%, p>0.05, n=6$, Fig. $7 G)$. Further, the magnitude of the flow/pressure-evoked vasoconstriction at the onset $(\Delta 22.23 \pm 3.33 \%$ vs $\Delta 23.50 \pm 4.60 \%, p>$ $0.05, n=6$; Fig. $7 H)$ or sustained phase $(\Delta 22.27 \pm 3.41 \%$ vs $\Delta 22.44 \pm 4.54 \%, p>0.05, n=6$; Fig. $7 H$ ) of the flow/pressure stimulus was unaffected by HET0016, suggesting a lack of contribution for 20-HETE signaling in flow/pressure-evoked increases in PA vascular tone.

Another recently proposed glial-derived vasoconstrictor is ATP (Kur and Newman, 2014). Given the ability of astrocytes to release ATP upon an increase in intracellular $\mathrm{Ca}^{2+}$ (Arcuino et al., 2002), we tested whether blockade of ATP signaling with the broad-spectrum P2 receptor antagonist suramin $(500 \mu \mathrm{mol} / \mathrm{L}$; 20-30 min perfusion) altered flow/pressure-evoked vascular responses in PAs. As with BAPTA, the presence of suramin significantly reduced resting PA tone $(\Delta 6.79 \pm 1.28 \%, p<0.05, n=$ 
A

$$
\text { Pial }
$$
Parenchymal arteriole arteriole
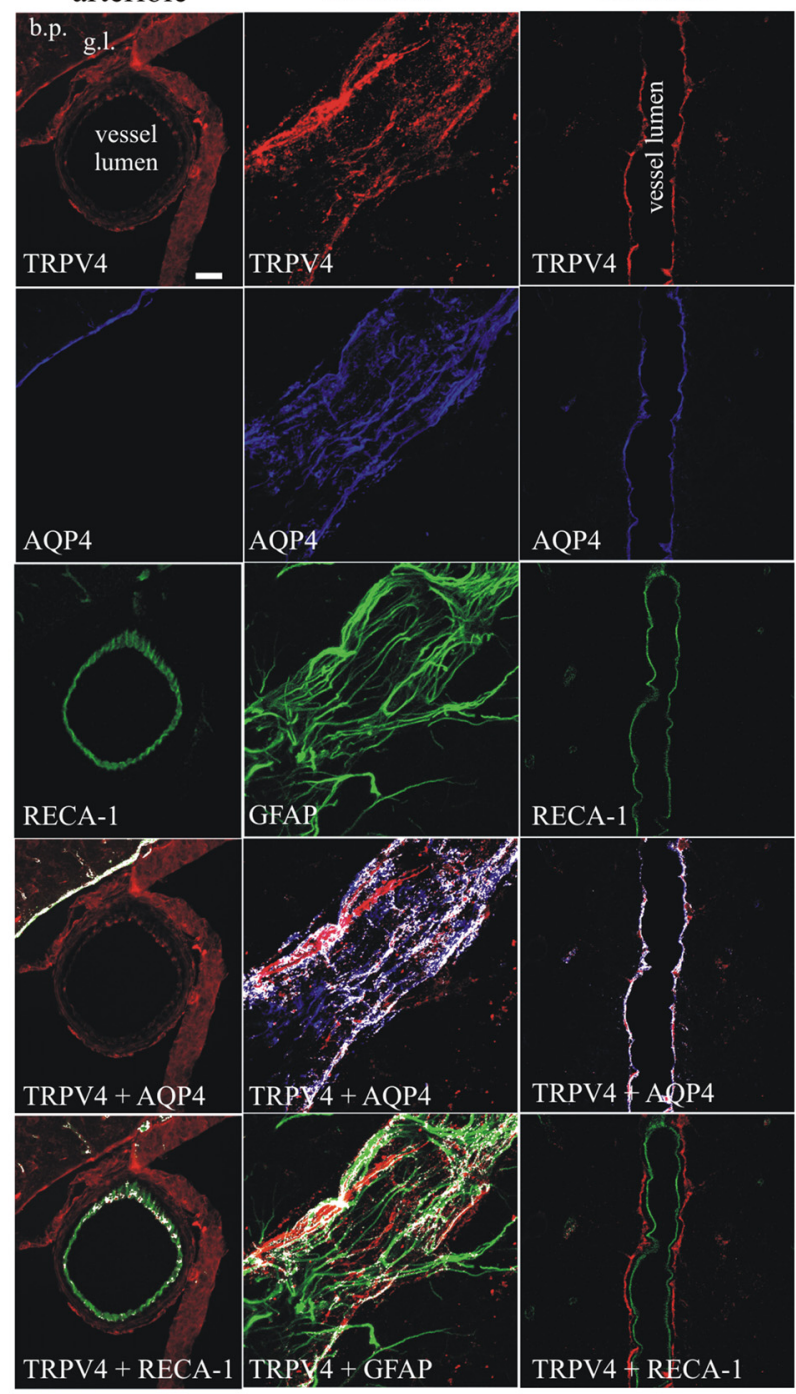

AQP

B
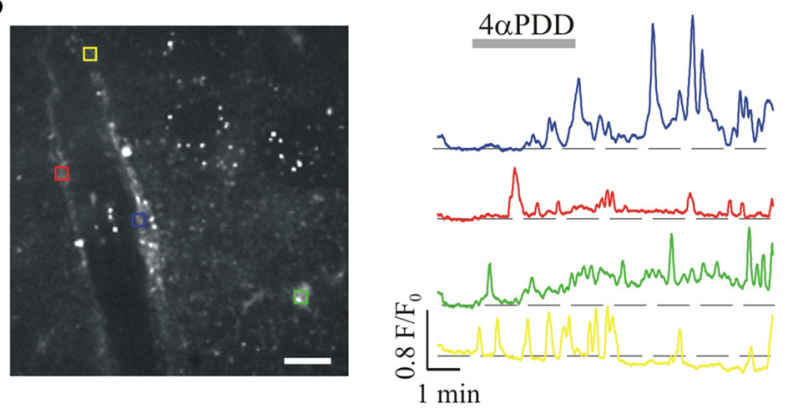

C
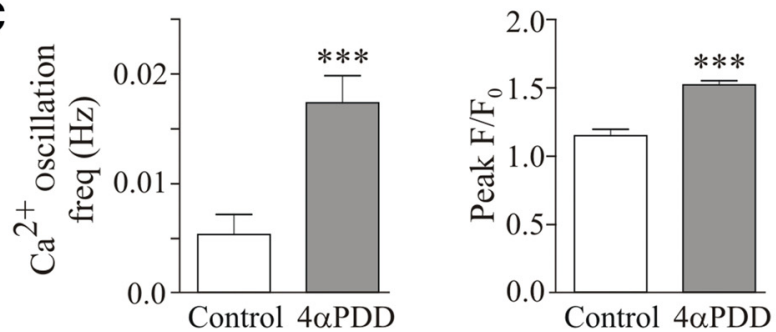

Figure 4. TRPV4 channel expression and function in cortical PAs. $\boldsymbol{A}$, Left column, Confocal images showing immunostaining for TRPV4 (red), the astrocytic endfoot marker AQP4 (blue), and the EC marker RECA-1 (green) in a pial arteriole (extraparenchymal vessel); note high
A

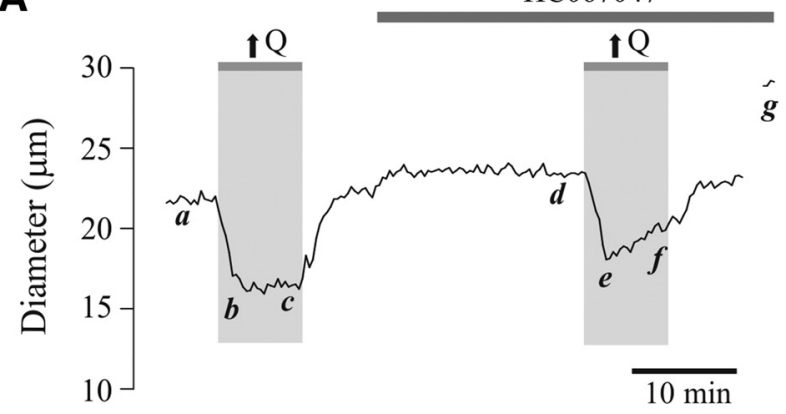

B

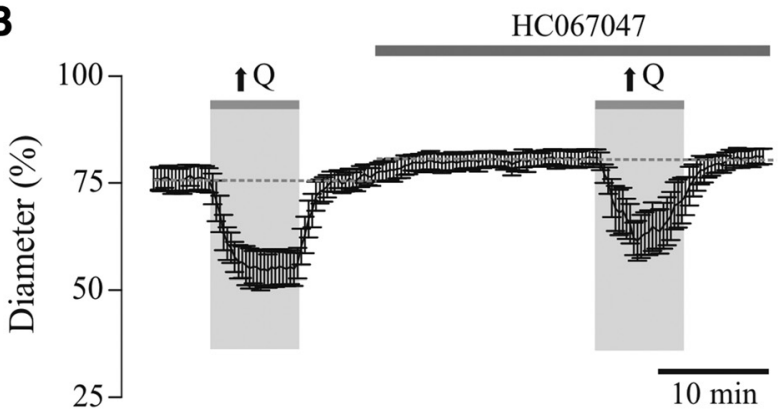

C

D
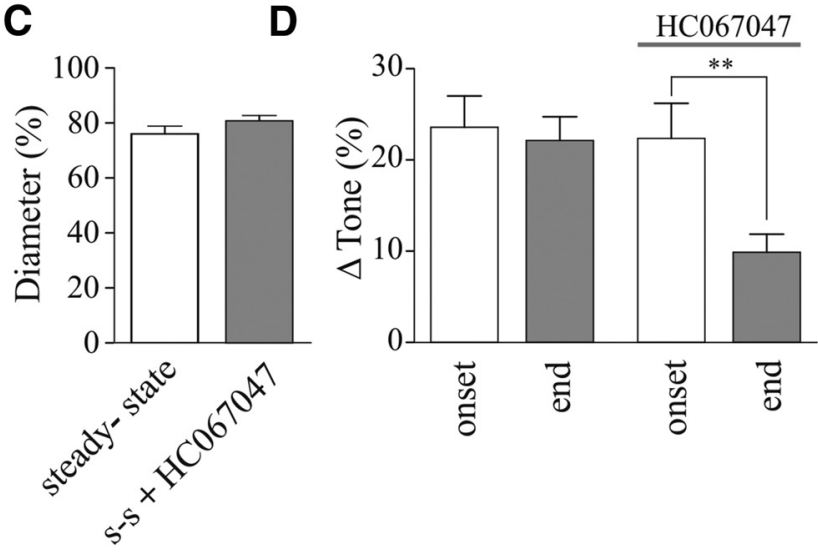

Figure 5. TRPV4 channel contribution to flow/pressure-evoked increases in PA tone. $\boldsymbol{A}$, Representative trace showing diameter changes in response to an increase in flow $(\uparrow Q)$ before and after bath application of the TRPV4 channel blocker, HC067047 [5 $\mu \mathrm{mol} / \mathrm{L} ; a$, d: steadystate $(s-s)$ tone; $b$, e: peak constriction at stimulus $(\uparrow Q)$ onset; $c$, $f$ : tone at the end of $\uparrow Q$ stimulus; $g$ : maximum diameter in zero $\mathrm{Ca}^{2+}+$ papaverine]. $\boldsymbol{B}$, Averaged diameter (percentage from maximum) changes in response to $\uparrow Q$.C , Averaged s-s tone (percentage from maximum) before and after $\mathrm{HC} 067047$ perfusion. $\boldsymbol{D}$, Averaged $\Delta$ tone (percentage) at the onset and end of the $\uparrow Q$ stimulus. ${ }^{* *} p<0.01$.

AQP4-TRPV4 colocalization along the glia limitans. Middle column, Confocal images showing immunostaining for TRPV4 (red), AQP4 (blue), and the astrocytic process marker GFAP (green) in a PA; images were acquired along the surface of the vessel to better visualize glial coverage. Right column, Confocal images showing immunostaining for TRPV4 (red), AQP4 (blue), and RECA-1 (green) in a PA; images were acquired along the midsection of the arteriole to appreciate TRPV4 colocalization with astrocytic endfeet and not ECs. Bottom two images for all three columns, White pixels denote colocalization (detected by a colocalization algorithm using ImageJ software). Scale bar, $25 \mu \mathrm{m}$. bp, Brain parenchyma; $g l$, glia limitans. B, Confocal image of Fluo4-loaded astrocytes with corresponding ROls (left; scale bar, $25 \mu \mathrm{m}$ ) and representative traces of astrocytic $\mathrm{Ca}^{2+}$ activity in response to the TRPV4 channel agonist $4 \alpha$ PDD (right, 5 $\mu \mathrm{mol} / \mathrm{L}$ ). C, Averaged astrocyte $\mathrm{Ca}^{2+}$ oscillation frequency (left) and peak $F / F_{0}$ amplitude (right) in response to $4 \alpha \mathrm{PDD} .{ }^{* * *} p<0.001$. 
A

$$
\text { A }
$$
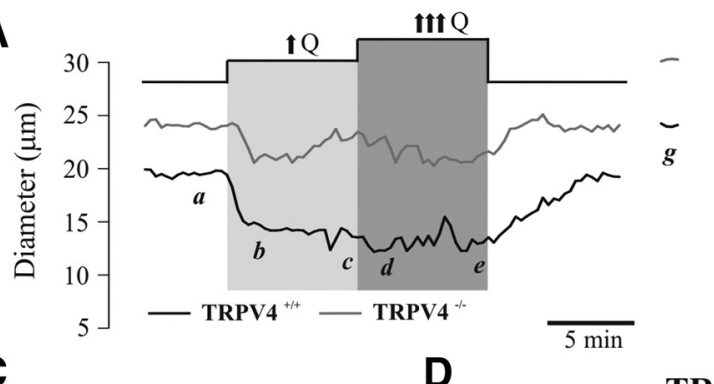

B

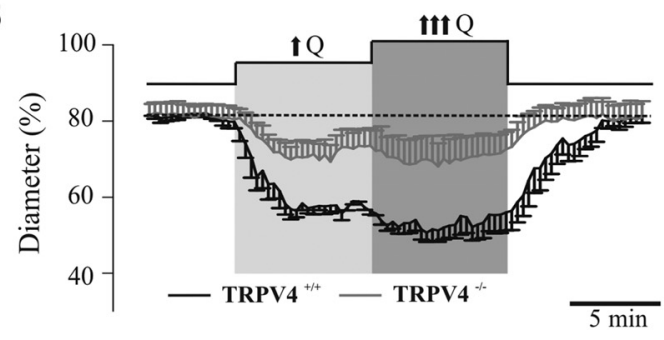

TRPV4 ${ }^{++1}$
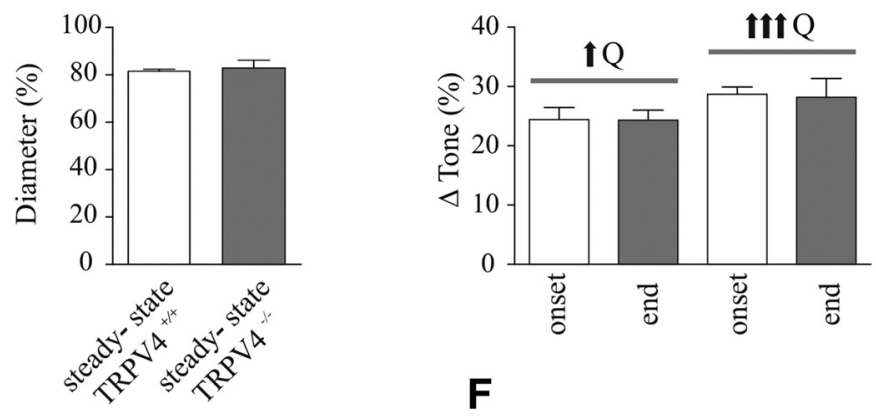

$\mathbf{F}$

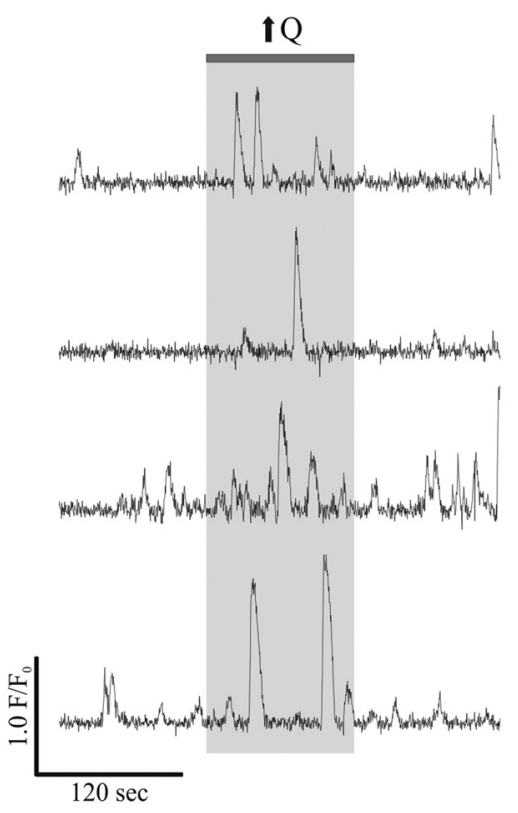

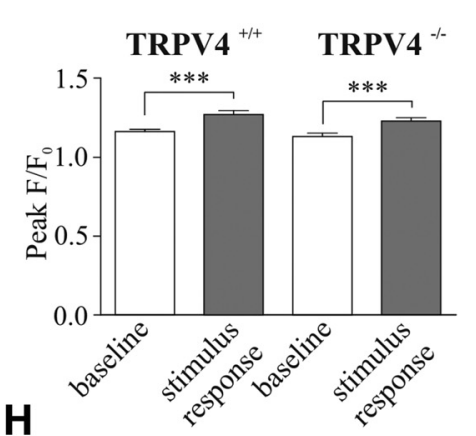

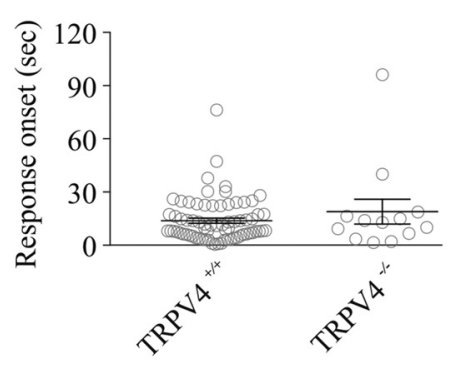

TRPV4 ${ }^{\text {- }}$

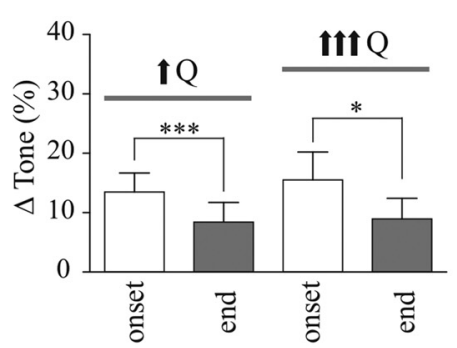

G
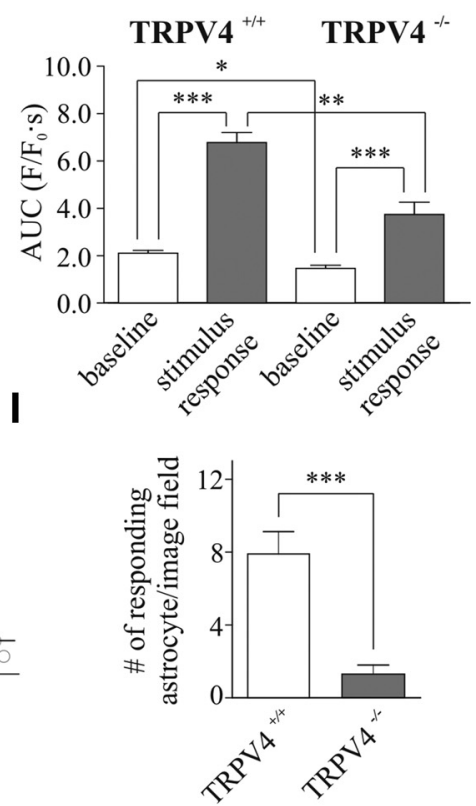

Figure 6. TRPV4 channel contribution to flow/pressure-evoked increases in PA tone and astrocyte activity. $\boldsymbol{A}$, Representative traces showing diameter changes in response to two incremental flow increases $(\uparrow Q, \uparrow \uparrow \uparrow Q)$ in TRPV4 ${ }^{+/+}$(black) and TRPV4 ${ }^{-1-}$ (gray) mice [a: steady-state (s-s) tone; $b, d$ : peak constriction at stimulus onset $(\uparrow Q, \uparrow \uparrow \uparrow \uparrow) ; c, e:$ tone at the end of the stimulus ( $\uparrow Q, \uparrow \uparrow \uparrow Q$ ); f: maximum diameter in zero $\mathrm{Ca}^{2+}+$ papaverine]. B. Averaged diameter (percentage from maximum) changes in response to $\uparrow Q$ or $\uparrow \uparrow \uparrow Q$ in TRPV4 ${ }^{+/+}$ (black) and TRPV4 ${ }^{-1-}$ (gray) mice. C, Averaged s-s tone (percentage from maximum) in TRPV4 ${ }^{+/+}$(left) and TRPV4 ${ }^{-1-}$ (right) mice. $\boldsymbol{D}$, Averaged $\Delta$ tone (percentage) at the onset and end of the $\uparrow Q$ or $\uparrow \uparrow \uparrow Q$ stimulus for TRPV4 ${ }^{+/+}$(left) and TRPV4 ${ }^{-/-}$(right) mice. $E$, Representative traces of astrocytic $\mathrm{Ca}^{2+}$ activity in response to $\uparrow$ Q from TRPV4 ${ }^{+/+}$mice. $F$, Averaged peak amplitude (peak $F / F_{0}$ ) at baseline (open bar) and stimulus response period (gray bar) from TRPV4 ${ }^{+/+}$(left) and TRPV4 ${ }^{-1-}$ (right) mice. G, Averaged AUC of baseline (open bar) and stimulus response period (gray bar) from TRPV4 ${ }^{+++}$(left) and TRPV4 ${ }^{-1-}$ (right) mice. $\boldsymbol{H}$, Onset time for astrocytic $\mathrm{Ca}^{2+}$ responses from TRPV4 ${ }^{+/+}$(left) and TRPV4 ${ }^{-1-}$ (right) mice. I, Averaged mean number of responding astrocytes to $\uparrow Q$ from TRPV4 ${ }^{+/+}$(left) and TRPV4 ${ }^{-1-}$ (right) mice. ${ }^{*} p<0.05,{ }^{* *} p<0.001$ and ${ }^{* * *} p<0.0001$.

10; Fig. $8 A-C)$. Moreover, as with the addition of BAPTA to the astrocyte syncytium or the TRPV4 channel blocker added to the bath, suramin significantly blunted the ability of PA to sustain vascular tone upon an increase in flow/pressure $(\Delta 16.34 \pm 2.02 \%$ vs $\Delta 6.70 \pm 1.40 \%, p<0.001, n=10$, data collected at minute 8 of the stimulus; Fig. $8 A, B, D)$. Increasing ATP concentration in astrocytes via the patch pipette resulted in a significant increase in the magnitude of the flow/pressure-evoked vasoconstriction response $(\Delta 39.47 \pm 2.56 \%$ vs $\Delta 23.59 \pm 2.85 \%$, $p=0.0017, n=4$; Fig. $8 E, G)$, although PA tone was not sustained throughout the stimulus period $(\Delta 0.36 \pm 0.08 \%$, $p=0.5926, n=4$; Fig. $8 E, G)$. Together these data support astrocyte-derived ATP as an important contributor to flow/ pressure-evoked vascular responses.

\section{Astrocytes rapidly sense flow/pressure changes in vivo: contribution of TRPV4 channels}

To test for vessel-to-astrocyte signaling in vivo along with TRPV4 channel contributions to resting $\mathrm{CBF}$ changes, we evaluated astrocytic responses to autoregulatory-mediated PA constriction 


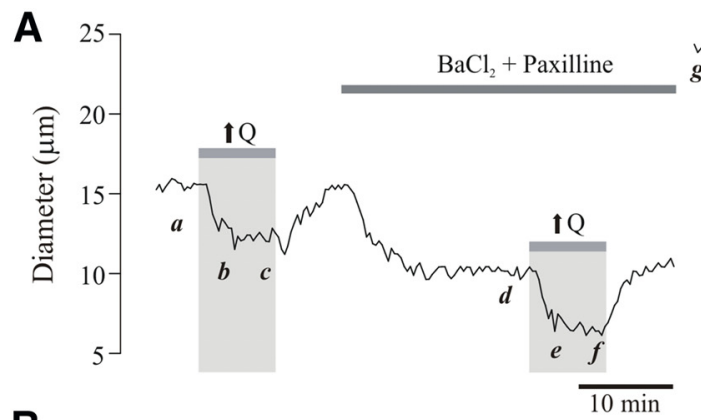

B

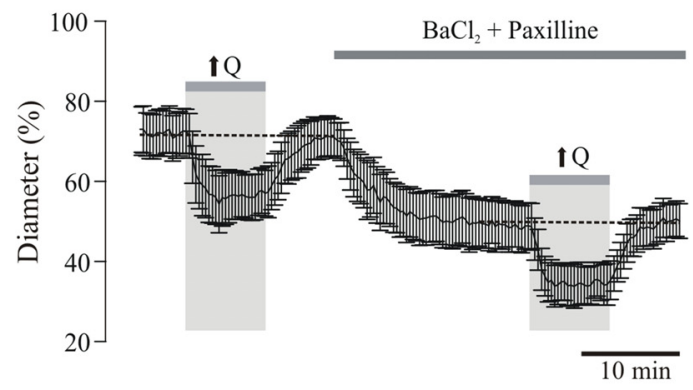

C

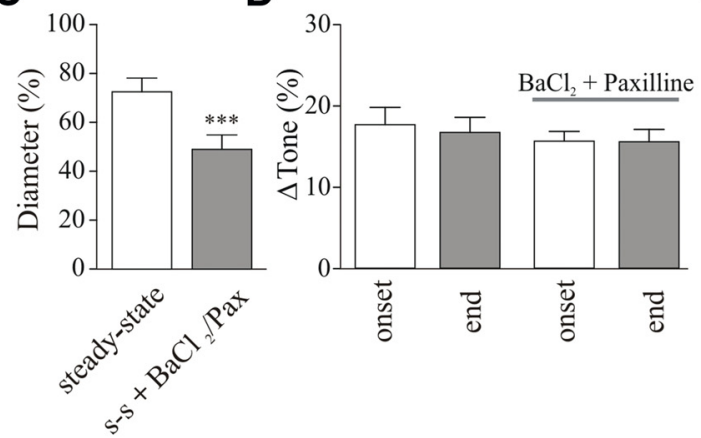

E

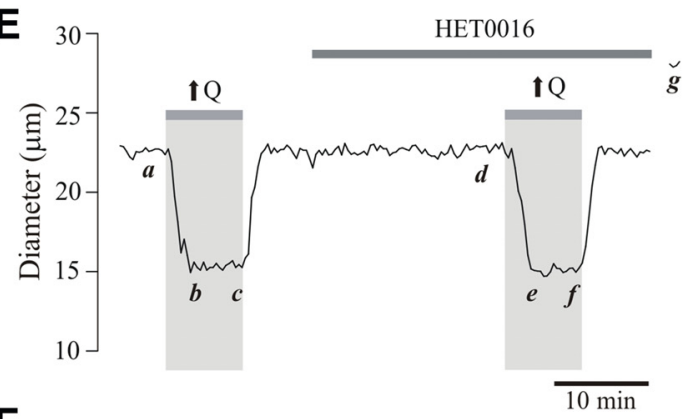

$\mathbf{F}$

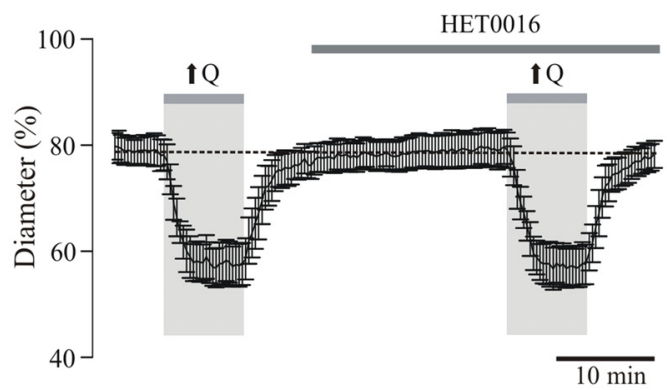

H

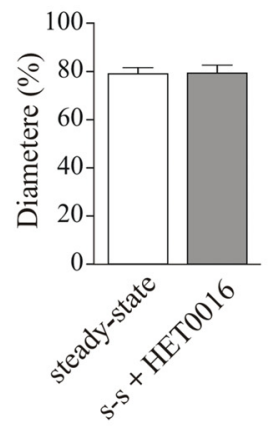

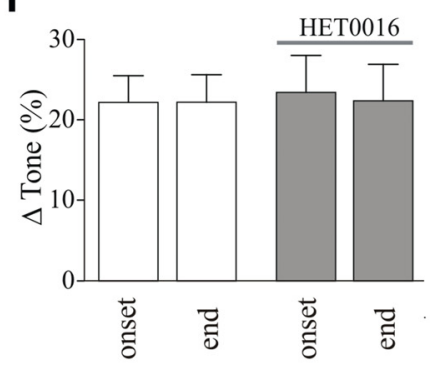

Figure 7. Flow/pressure-evoked PA constriction is not mediated by $\mathrm{K}^{+}$or 20 -HETE signaling. $A$, Representative trace showing diameter changes in response to flow increases ( $\uparrow Q$ ) before and after bath application of the $\mathrm{K}^{+}$channel blockers, $\mathrm{BaCl}_{2}(100 \mu \mathrm{mol} / \mathrm{L})$ and paxilline $[2 \mu \mathrm{mol} / \mathrm{L} ; a$, d: steady-state $(s-s)$ tone; $b$, e: peak constriction at stimulus $(\uparrow Q)$ onset; $c, f$ : tone at the end of $\uparrow Q$ stimulus; $g$ : maximum diameter in zero $\mathrm{Ca}^{2+}+$ papaverine]. $\boldsymbol{B}$, Averaged diameter (percentage from maximum) changes in response to $\uparrow Q$ before and after $\mathrm{BaCl}_{2}$ and paxilline perfusion. C, Averaged s-s tone (percentage from maximum) before and after $\mathrm{BaCl}_{2}$ and paxilline perfusion. $\boldsymbol{D}$, Averaged $\Delta$ tone (percentage) at the onset and end of the $\uparrow Q$ stimulus. $\boldsymbol{E}$, Representative trace showing diameter changes in response to $\uparrow Q$ before and after bath application of HET0016 $[100 \mathrm{nmol} / L ; a, d: s$-s tone; $b$, e: peak constriction at stimulus ( $\uparrow Q$ ) onset; $c$, $f$ : tone at the end of $\uparrow Q$ stimulus; $g$ : maximum diameter in zero $\mathrm{Ca}^{2+}+$ papaverine]. $\boldsymbol{F}$, Averaged diameter (percentage from maximum) changes in response to $\uparrow$ Q before and after HET0016 perfusion. G, Summary data showing s-s tone (percentage) before and after HET0016 perfusion. $\boldsymbol{H}$, Averaged $\Delta$ tone (percentage) at the onset and end of the $\uparrow Q$ stimulus. ${ }^{* * *} p<0.001$.

induced by a sudden increase in mean arterial pressure (MAP) in $\mathrm{TRPV}^{+/+}$and TRPV4 ${ }^{-1-}$ mice. Systemic MAP was increased by a bolus tail vein injection of Phe (5-20 $\mu \mathrm{g} / \mathrm{kg}$; Niwa et al., 2002; Ayata et al., 2004; Lu et al., 2009). MAP was assessed indirectly by measuring the corresponding baroreflex-mediated drop in HR. Astrocytic $\mathrm{Ca}^{2+}$ responses were measured simultaneously with HR. In TRPV $4^{+/+}$mice, Phe induced a rapid baroreflexmediated drop in HR $[\Delta-174.84 \pm 29.58$ beats per minute (bpm), $n=8]$, which coincided with a significant increase in astrocytic $\mathrm{Ca}^{2+}\left(\Delta F / F_{0} 0.78 \pm 0.23, p=0.01, n=8\right.$ from 133 astrocytes; Fig. $9 A-D$ ). As shown in Figure $6 I$, in TRPV $4^{-1-}$ mice, the mean number of responding astrocytes to Phe injection was significantly reduced compared with TRPV $4^{+/+}$mice $(16.63 \pm 1.63$ in $\mathrm{TRPV}^{+/+}$vs $8.33 \pm 1.05$ in TRPV $^{-/-}$mice, $p=0.002$; Fig. $9 B)$. However, the mean drop in $\mathrm{HR}(\Delta-202.46 \pm 42.21 \mathrm{bpm}, n=$ 6) and mean increase in astrocytic $\Delta \mathrm{Ca}^{2+}\left(\Delta F / F_{0} 0.44 \pm 0.15\right.$, $n=6$ from 50 astrocytes, $p=0.03$ ) were not different from those observed in TRPV $4^{+/+}$mice (Fig. 9C,D).

\section{Discussion}

In this study, we provide evidence for a novel function of astrocytes, in which these cells contribute to PA hemodynamic-evoked changes in vascular tone. Our data support a previously undefined signaling mechanism in the neurovascular unit, in which information flows in the reverse direction (i.e., vessel to astrocyte) as that previously described for NVC. By measuring concomitant changes in VSMC and astrocytic $\mathrm{Ca}^{2+}$ in brain slices, we demonstrate that astrocytes are activated by hemodynamicevoked changes in perfused and pressurized PAs. Experiments addressing the time course of this novel form of communication (Fig. 2) support a sequential activation of cells from vessel to astrocyte. Moreover, we identify mechanosensitive TRPV4 channels expressed in astrocytes as key molecular contributors to PA tone and show that their selective pharmacological inhibition or genetically encoded absence impairs glial $\mathrm{Ca}^{2+}$ responses during hemodynamic changes. Finally, we propose ATP as a putative glial-derived signal contributing to the regulation of PA tone 

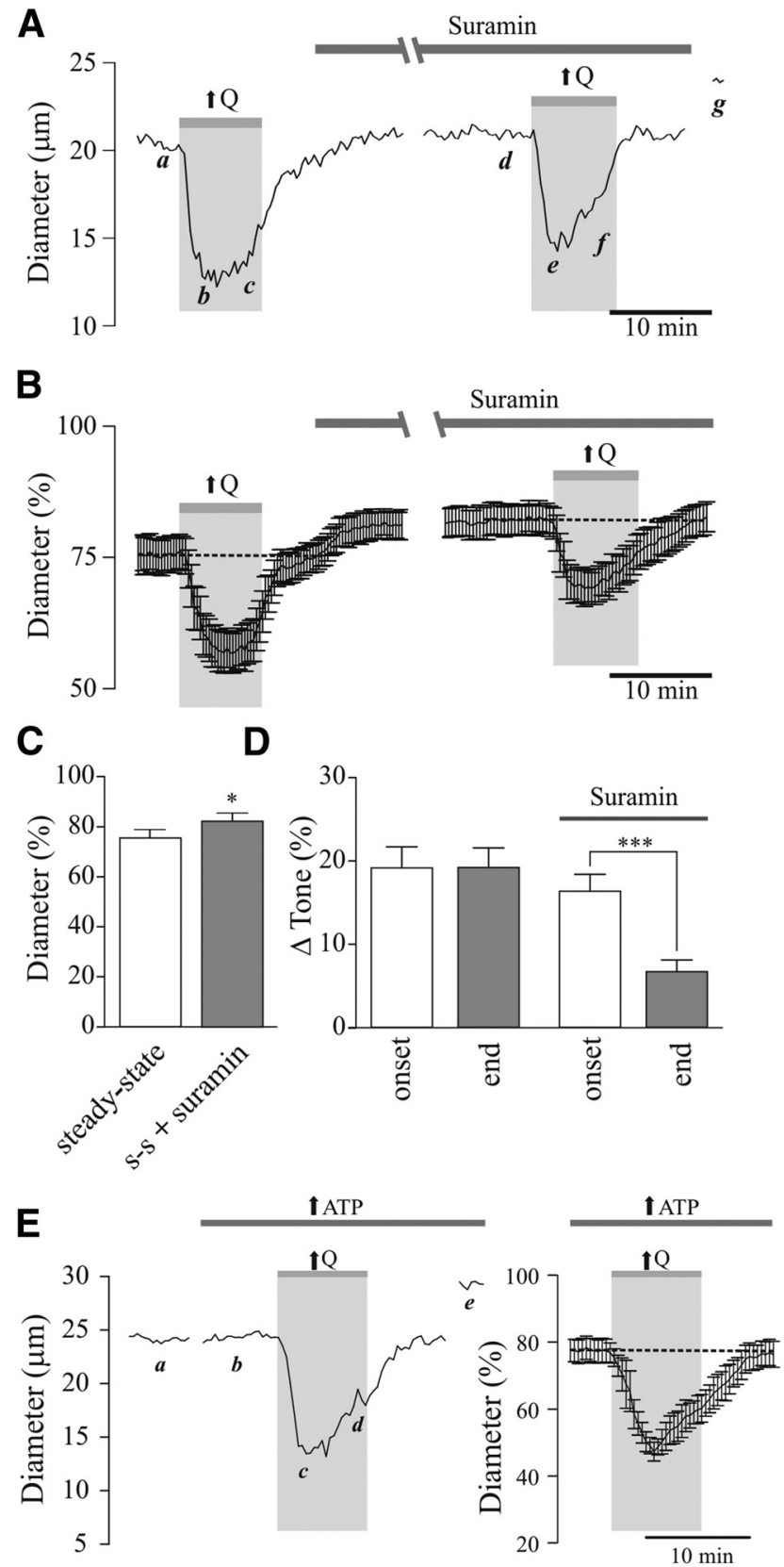

$\mathbf{F}$
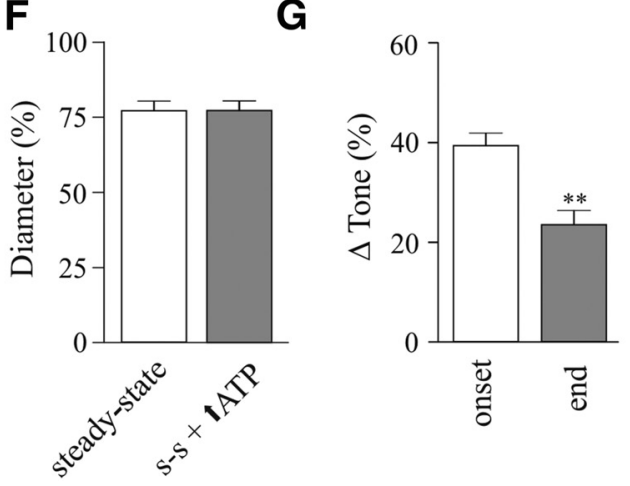

Figure 8. Contribution of purinergic signaling to flow/pressure-evoked increases in PA tone. $\boldsymbol{A}$, Representative trace showing diameter changes in response to flow increases $(\uparrow Q$ ) before and after bath application of suramin $[500 \mu \mathrm{mol} / \mathrm{L} ; a, d$ : steady-state $(s-s)$ tone; $b, e$ : peak constriction at stimulus ( $\uparrow Q$ ) onset; $c$, $f$ : tone at the end of $\uparrow Q$ stimulus; $g$ : maximum diameter in zero $\mathrm{Ca}^{2+}+$ papaverine]. $\boldsymbol{B}$, Averaged diameter (percentage from maximum) changes following flow/pressure-induced $\mathrm{Ca}^{2+}$ activation in astrocytes. Together, our results support a novel bidirectional signaling modality within the neurovascular unit in which the astrocyte serves as a mechanosensory system to dynamically modulate steadystate vascular responses.

Using an innovative in vitro brain slice approach that allows perfusion of PAs, we demonstrate that astrocytes transduce PA hemodynamic changes into increased intracellular $\mathrm{Ca}^{2+}$ levels. Moreover, results showing blunted PA myogenic tone and decreased ability of PAs to sustain increases in tone during a hemodynamic challenge upon chelation of astrocytic $\mathrm{Ca}^{2+}$ suggest that astrocytes are active regulators of basal cerebrovascular tone and can also modulate the magnitude and duration of flow/pressureevoked vasoconstrictions. Consistent with the notion that TRPV4 channels serve as the hemodynamic sensor and transducer and corroborating previous findings (Benfenati et al., 2007, 2011; Dunn et al., 2013), we find TRPV4 channels to be strategically expressed on the adventitial side of PAs, where they colocalize with astrocytic endfeet and processes. Conversely, and in contrast to pial arterioles (Earley et al., 2005; Marrelli et al., 2007; Zhang et al., 2013), we show that TRPV4 channel expression is not evident in the endothelium of PAs, further supporting a role for astrocytic TRPV4 channels in hemodynamic-induced changes in vascular tone. A few studies have shown that TRPV4 channels expressed in ECs from pial arterioles and VSMCs contribute to vasodilation (Earley et al., 2005; Köhler et al., 2006; Hartmannsgruber et al., 2007; Mendoza et al., 2010). However, we show that flow/pressure-evoked vasoconstrictions in TRPV $4^{-I-}$ mice are significantly decreased compared with those in TRPV4 ${ }^{+/+}$mice (Fig. 6A, B,D). In support of our structural data, pharmacological activation of these channels induced similar astrocytic $\mathrm{Ca}^{2+}$ responses to those observed during flow/pressure-evoked changes in vascular tone. Moreover, pharmacological blockade of TRPV4 channels abrogated the ability of PAs to sustain flow/pressureevoked PA constrictions. Of caution is the fact that bath application of TRPV4 channel blocker will target TRPV4 channels expressed in other cell types, such as microglia (Konno et al., 2012) and neurons (Lee and Choe, 2014). However, the observations obtained from the BAPTA experiments (Fig. 3) along with those from the TRPV4 ${ }^{-1-}$ mice (Fig. 6) support a role for astrocyte TRPV4 channels in the regulation of PA tone during flow/pressure-evoked changes.

The mechanism underlying flow/pressure-evoked TRPV4 channel activation is not clear. Flow/pressure-evoked vasoconstriction increases shear stress, which has been shown previously to activate TRPV4 channels in human embryonic kidney 293 (Gao et al., 2003). Additionally, because increases in flow/pressure in PA induced arteriole constriction, it is not clear whether it is shear or a change in the arteriole membrane (and concomitant stretching of the astrocyte membrane) that activates TRPV4 channels in astrocytes. On the other hand, and based on previous studies, it is also possible that a chemical pathway is also at play. Increased EC shear stress results in the release of arachidonic acid (AA) and downstream eicosanoids, such as epoxyeicosatrienoic

in response to $\uparrow Q$ before and after suramin perfusion. $C$, Averaged $s$-s tone (percentage from maximum) before and after suramin perfusion. $\boldsymbol{D}$, Averaged $\Delta$ tone (percentage) at the onset and end of the $\uparrow Q$ stimulus. $\boldsymbol{E}$, Representative trace (left) and average diameter (right) changes in response to $\uparrow Q$, before and after high ATP loading into the astrocytic syncytium $(a$, $b$ : s-s tone; $c$ : peak constriction at stimulus ( $\uparrow Q$ ) onset; $d$ : tone at the end of $\uparrow Q$ stimulus; $e$ : maximum diameter in zero $\mathrm{Ca}^{2+}+$ papaverine). $\boldsymbol{F}$, Averaged s-s tone (percentage from maximum) before and after suramin perfusion. $G$, Averaged $\Delta$ tone (percentage) at the onset and end of the $\uparrow Q$ stimulus. ${ }^{*} p<0.05,{ }^{* *} p<0.01$. 


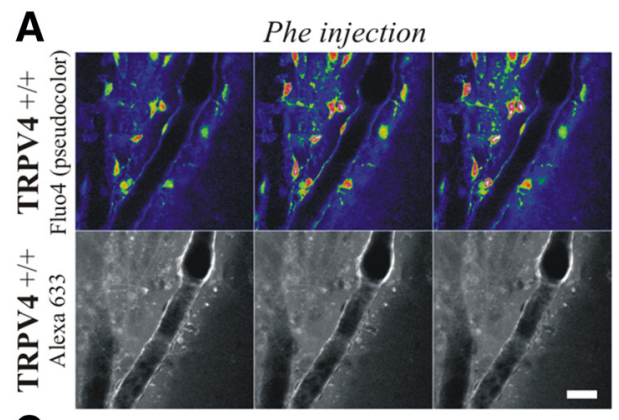

C

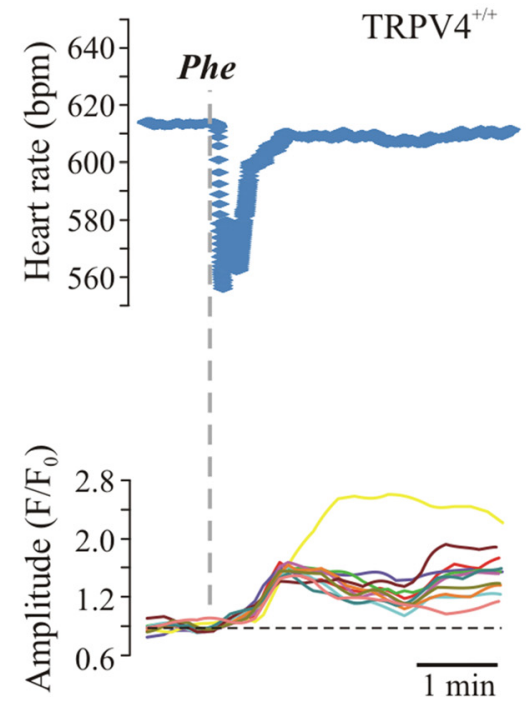

B
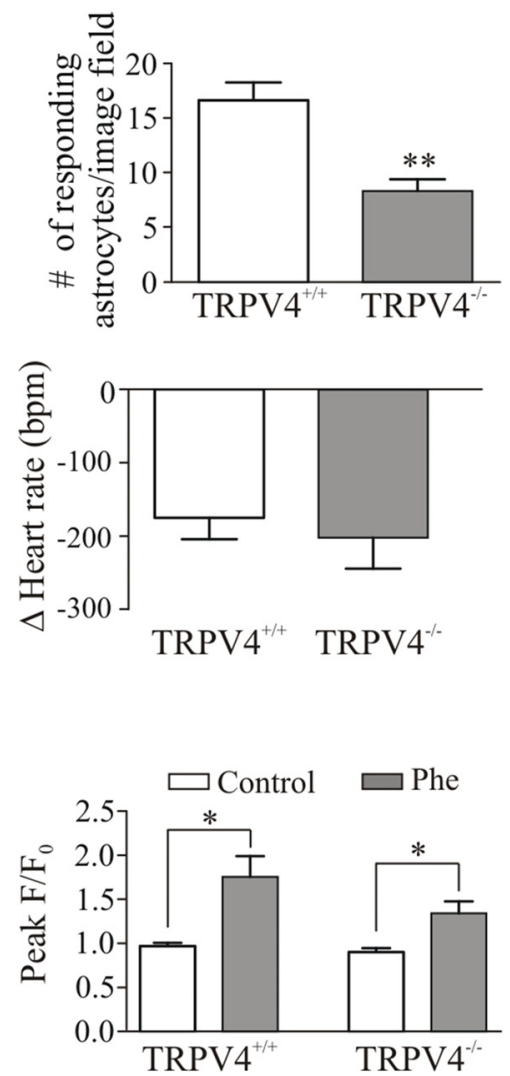

astrocyte-derived ATP as a putative signal contributing to the sustained phase of flow/ pressure-induced vasoconstriction. This idea is supported by studies in the retina, where astrocyte-derived ATP was shown to tonically constrict arterioles via $\mathrm{P} 2 \mathrm{X} 1$ receptor activation (Kur and Newman, 2014). However, given that suramin is a broadspectrum purinergic receptor blocker, other purinergic signaling mechanisms cannot be ruled out. These include P2Y receptors, which Brayden et al. (2013) showed to contribute to PA myogenic tone in excised PA (which lack surrounding astrocytes). Thus, further studies will be needed to determine the identity of the purinergic receptor subtype involved.

Using a well established systemic challenge paradigm, we also tested whether astrocytic responses to vascular hemodynamic challenges occur in vivo. Our results show that an acute and transient systemic Phe injection, which increases MAP and evokes CA (i.e., arteriole vasoconstriction; Niwa et al., 2002; Ayata et al., 2004), increases astrocytic $\mathrm{Ca}^{2+}$ comparable to observations in vitro, further supporting the reverse flow of information within the neurovascular unit. The slow temporal resolution in our in vivo approach, however, failed to conclusively demonstrate the precise onset of astrocyte activation. Moreover, the transient nature of the stimulus (Phe injection) prevented our ability to assess more sustained responses. Thus, while our in vivo studies conclusively demonstrate the ability of astrocytes to respond to a hemodynamic challenge, they could not efficiently deter-

Figure 9. TRPV4 channels contribute to flow/pressure-induced astrocyte responses in vivo. $A, 2$ PLMS images from a TRPV $4^{+/+}$ mouse showing astrocytic $\mathrm{Ca}^{2+}$ activity (top) and the lining of the arteriole (bottom) following a systemic Phe injection. $\boldsymbol{B}$, Summary data showing number of responding astrocytes following systemic Phe injection in TRPV $4^{+/+}$and TRPV $4^{-/-}$mice. $\boldsymbol{C}$, Representative trace showing changes in HR in response to Phe injection in a TRPV4 ${ }^{+/+}$mouse (left); summary data of $\Delta$ HR in TRPV4 $^{+/+}$and TRPV4 ${ }^{-1-}$ mice (right). D , Representative trace showing changes in astrocytic $\mathrm{Ca}^{2+}$ activity in response to Phe injection in a TRPV4 ${ }^{+/+}$mouse (left); summary data showing changes in $\mathrm{Ca}^{2+}$ oscillation peak $F / F_{0}$ amplitude in TRPV4 ${ }^{+/+}$and TRPV4 ${ }^{-1-}$ mice (right). ${ }^{*} p<0.05,{ }^{* *} p<0.01$.

acids (EETs). While AA does not directly activate TRVP4 channels (it requires metabolism; Watanabe et al., 2003), EETs are a downstream putative endogenous TRPV4 channel agonist (Watanabe et al., 2003; Dunn et al., 2013). In support of this idea we previously showed that EETs mediated biphasic PA vascular responses and increased $\mathrm{Ca}^{2+}$ activity in astrocytes (Blanco et al., 2008). Further studies are needed to better address targeted astrocyte channels/receptors during increases in flow/pressure within PA. Moreover, as with NVC, it is likely that multiple potential signals act in concert to modulate hemodynamic-evoked PA responses.

Importantly, hemodynamic stimuli not only induced a change in astrocytic $\mathrm{Ca}^{2+}$ activity, but also triggered astrocyte participation in PA vascular responses, specifically during the sustained phase of the vasoconstrictive response. Both $\mathrm{K}^{+}$and 20-HETE were ruled out as potential glial-derived signals in this process, as blockade of these signaling pathways had no effect on the properties of hemodynamic-induced vascular responses. However, given previous studies on $\mathrm{K}^{+}$and 20-HETE signaling, it is possible that a given level of astrocytic $\mathrm{Ca}^{2+}$ is needed to induce sufficient $\mathrm{K}^{+}$efflux (Girouard et al., 2010) and/or 20-HETE formation (Mulligan and MacVicar, 2004). On the other hand, the purinergic receptor blocker suramin blunted the capacity of PAs to maintain flow/pressure-evoked constrictions. These data suggest mine their contribution to the sustained component of the flow/ pressure-evoked vasoconstriction response as evaluated in vitro. Consistent with an important role for TRPV4 channels in vesselto-astrocyte communication, both in vitro and in vivo studies showed a significant decrease in the number of responding astrocytes in the TRPV4 ${ }^{-1-}$ mouse. The partial persistence of astrocytic $\mathrm{Ca}^{2+}$ responses in TRPV4 ${ }^{-1-}$ mice may be due to the fact that TRPV4 channels can heteromerize with TRPC1 (Ma et al., 2011) or TRPP2 (Köttgen et al., 2008) channels to form functional channels. Alternatively, and, as with NVC mechanisms, multiple other channels/receptors may contribute to this process. While development of a glia-specific TRPV4 channel knock-out would be needed to more conclusively determine the role of glial TRPV4 channels in vessel-to-astrocyte signaling, our data support astrocytic TRPV4 channels as key contributors to astrocytemediated regulation of PA vascular tone in response to flow/ pressure changes.

In summary, we show that flow/pressure-evoked PA vasoconstriction (Kim and Filosa, 2012) activates $\mathrm{Ca}^{2+}$ signaling in cortical astrocytes, which in turn contributes to the sustained phase of the vasosconstriction response. In addition, we present evidence indicating that astrocyte TRPV4 channels and ATP are key players in this novel bidirectional vessel-to-astrocyte communication loop, contributing to adaptive myogenic tone adjust- 
ments, such as those occurring during CA. Based on previous NVC studies (Zonta et al., 2003; Mulligan and MacVicar, 2004; Filosa et al., 2006; Metea and Newman, 2006; Girouard et al., 2010) and the results from the present study, we propose that astrocytes are critical intermediaries regulating the flow of information between neurons and the local microvasculature in a bidirectional manner (Filosa and Iddings, 2013). Further, we speculate that this newly discovered signaling modality serves as a mechanosensory system that dynamically modulates vascular tone according to the status of PA perfusion pressure, providing evidence for an active role for astrocytes in the process of CA.

\section{References}

Arcuino G, Lin JH, Takano T, Liu C, Jiang L, Gao Q, Kang J, Nedergaard M (2002) Intercellular calcium signaling mediated by point-source burst release of ATP. Proc Natl Acad Sci U S A 99:9840-9845. CrossRef Medline

Attwell D, Buchan AM, Charpak S, Lauritzen M, Macvicar BA, Newman EA (2010) Glial and neuronal control of brain blood flow. Nature 468:232243. CrossRef Medline

Ayata C, Dunn AK, Gursoy-OZdemir Y, Huang Z, Boas DA, Moskowitz MA (2004) Laser speckle flowmetry for the study of cerebrovascular physiology in normal and ischemic mouse cortex. J Cereb Blood Flow Metab 24:744-755. CrossRef Medline

Bagi Z, Ungvari Z, Szollár L, Koller A (2001) Flow-induced constriction in arterioles of hyperhomocysteinemic rats is due to impaired nitric oxide and enhanced thromboxane A(2) mediation. Arterioscler Thromb Vasc Biol 21:233-237. CrossRef Medline

Benfenati V, Amiry-Moghaddam M, Caprini M, Mylonakou MN, Rapisarda C, Ottersen OP, Ferroni S (2007) Expression and functional characterization of transient receptor potential vanilloid-related channel 4 (TRPV4) in rat cortical astrocytes. Neuroscience 148:876-892. CrossRef Medline

Benfenati V, Caprini M, Dovizio M, Mylonakou MN, Ferroni S, Ottersen OP, Amiry-Moghaddam M (2011) An aquaporin-4/transient receptor potential vanilloid 4 (AQP4/TRPV4) complex is essential for cell-volume control in astrocytes. Proc Natl Acad Sci U S A 108:2563-2568. CrossRef Medline

Blanco VM, Stern JE, Filosa JA (2008) Tone-dependent vascular responses to astrocyte-derived signals. Am J Physiol Heart Circ Physiol 294:H2855H2863. CrossRef Medline

Brayden JE, Earley S, Nelson MT, Reading S (2008) Transient receptor potential (TRP) channels, vascular tone and autoregulation of cerebral blood flow. Clin Exp Pharmacol Physiol 35:1116-1120. CrossRef Medline

Brayden JE, Li Y, Tavares MJ (2013) Purinergic receptors regulate myogenic tone in cerebral parenchymal arterioles. J Cereb Blood Flow Metab 33: 293-299. CrossRef Medline

Bryan RM Jr, Marrelli SP, Steenberg ML, Schildmeyer LA, Johnson TD (2001) Effects of luminal shear stress on cerebral arteries and arterioles. Am J Physiol Heart Circ Physiol 280:H2011-H2022. Medline

Cauli B, Hamel E (2010) Revisiting the role of neurons in neurovascular coupling. Front Neuroenergetics 2:9. CrossRef Medline

Chan SL, Sweet JG, Cipolla MJ (2013) Treatment for cerebral small vessel disease: effect of relaxin on the function and structure of cerebral parenchymal arterioles during hypertension. FASEB J 27:3917-3927. CrossRef Medline

Cipolla MJ, Smith J, Kohlmeyer MM, Godfrey JA (2009) SKCa and IKCa channels, myogenic tone, and vasodilator responses in middle cerebral arteries and parenchymal arterioles: effect of ischemia and reperfusion. Stroke 40:1451-1457. CrossRef Medline

Duling BR, Gore RW, Dacey RG Jr, Damon DN (1981) Methods for isolation, cannulation, and in vitro study of single microvessels. Am J Physiol 241:H108-H116. Medline

Dunn KM, Hill-Eubanks DC, Liedtke WB, Nelson MT (2013) TRPV4 channels stimulate $\mathrm{Ca}^{2+}$-induced $\mathrm{Ca}^{2+}$ release in astrocytic endfeet and amplify neurovascular coupling responses. Proc Natl Acad Sci U S A 110: 6157-6162. CrossRef Medline

Earley S, Heppner TJ, Nelson MT, Brayden JE (2005) TRPV4 forms a novel $\mathrm{Ca}^{2+}$ signaling complex with ryanodine receptors and BKCa channels. Circ Res 97:1270-1279. CrossRef Medline

Earley S, Pauyo T, Drapp R, Tavares MJ, Liedtke W, Brayden JE (2009)
TRPV4-dependent dilation of peripheral resistance arteries influences arterial pressure. Am J Physiol Heart Circ Physiol 297:H1096-H1102. CrossRef Medline

Faraci FM, Baumbach GL, Heistad DD (1989) Myogenic mechanisms in the cerebral circulation. J Hypertens Suppl 7:S61-S64; discussion S65. Medline

Félétou M, Huang Y, Vanhoutte PM (2011) Endothelium-mediated control of vascular tone: COX-1 and COX-2 products. Br J Pharmacol 164:894912. CrossRef Medline

Félétou M, Köhler R, Vanhoutte PM (2012) Nitric oxide: orchestrator of endothelium-dependent responses. Ann Med 44:694-716. CrossRef Medline

Filosa JA, Iddings JA (2013) Astrocyte regulation of cerebral vascular tone. Am J Physiol Heart Circ Physiol 305:H609-H619. CrossRef Medline

Filosa JA, Bonev AD, Straub SV, Meredith AL, Wilkerson MK, Aldrich RW, Nelson MT (2006) Local potassium signaling couples neuronal activity to vasodilation in the brain. Nat Neurosci 9:1397-1403. CrossRef Medline

Gao X, Wu L, O’Neil RG (2003) Temperature-modulated diversity of TRPV4 channel gating: activation by physical stresses and phorbol ester derivatives through protein kinase $\mathrm{C}$-dependent and -independent pathways. J Biol Chem 278:27129-27137. CrossRef Medline

Girouard H, Bonev AD, Hannah RM, Meredith A, Aldrich RW, Nelson MT (2010) Astrocytic endfoot $\mathrm{Ca}^{2+}$ and BK channels determine both arteriolar dilation and constriction. Proc Natl Acad Sci U S A 107:3811-3816. CrossRef Medline

Harder DR, Narayanan J, Gebremedhin D (2011) Pressure-induced myogenic tone and role of 20-HETE in mediating autoregulation of cerebral blood flow. Am J Physiol Heart Circ Physiol 300:H1557-H1565. CrossRef Medline

Hartmannsgruber V, Heyken WT, Kacik M, Kaistha A, Grgic I, Harteneck C, Liedtke W, Hoyer J, Köhler R (2007) Arterial response to shear stress critically depends on endothelial TRPV4 expression. PLoS One 2:e827. CrossRef Medline

Kacem K, Lacombe P, Seylaz J, Bonvento G (1998) Structural organization of the perivascular astrocyte endfeet and their relationship with the endothelial glucose transporter: a confocal microscopy study. Glia 23:1-10. CrossRef Medline

Kennelly SP, Lawlor BA, Kenny RA (2009) Blood pressure and dementia-a comprehensive review. Ther Adv Neurol Disord 2:241-260. CrossRef Medline

Kim KJ, Filosa JA (2012) Advanced in vitro approach to study neurovascular coupling mechanisms in the brain microcirculation. J Physiol 590: 1757-1770. CrossRef Medline

Köhler R, Heyken WT, Heinau P, Schubert R, Si H, Kacik M, Busch C, Grgic I, Maier T, Hoyer J (2006) Evidence for a functional role of endothelial transient receptor potential V4 in shear stress-induced vasodilatation. Arterioscler Thromb Vasc Biol 26:1495-1502. CrossRef Medline

Koller A, Toth P (2012) Contribution of flow-dependent vasomotor mechanisms to the autoregulation of cerebral blood flow. J Vasc Res 49:375389. CrossRef Medline

Konno M, Shirakawa H, Iida S, Sakimoto S, Matsutani I, Miyake T, Kageyama K, Nakagawa T, Shibasaki K, Kaneko S (2012) Stimulation of transient receptor potential vanilloid 4 channel suppresses abnormal activation of microglia induced by lipopolysaccharide. Glia 60:761-770. CrossRef Medline

Köttgen M, Buchholz B, Garcia-Gonzalez MA, Kotsis F, Fu X, Doerken M, Boehlke C, Steffl D, Tauber R, Wegierski T, Nitschke R, Suzuki M, Kramer-Zucker A, Germino GG, Watnick T, Prenen J, Nilius B, Kuehn EW, Walz G (2008) TRPP2 and TRPV4 form a polymodal sensory channel complex. J Cell Biol 182:437-447. CrossRef Medline

Kur J, Newman EA (2014) Purinergic control of vascular tone in the retina. J Physiol 592:491-504. CrossRef Medline

Lee JC, Choe SY (2014) Age-related changes in the distribution of transient receptor potential vanilloid 4 channel (TRPV4) in the central nervous system of rats. J Mol Histol 45:497-505. CrossRef Medline

Lu Y, Ma X, Sabharwal R, Snitsarev V, Morgan D, Rahmouni K, Drummond HA, Whiteis CA, Costa V, Price M, Benson C, Welsh MJ, Chapleau MW, Abboud FM (2009) The ion channel ASIC2 is required for baroreceptor and autonomic control of the circulation. Neuron 64:885-897. CrossRef Medline

Ma X, Nilius B, Wong JW, Huang Y, Yao X (2011) Electrophysiological 
properties of heteromeric TRPV4-C1 channels. Biochim Biophys Acta 1808:2789-2797. CrossRef Medline

Marrelli SP, O'Neil RG, Brown RC, Bryan RM Jr (2007) PLA2 and TRPV4 channels regulate endothelial calcium in cerebral arteries. Am J Physiol Heart Circ Physiol 292:H1390-H1397. Medline

Mendoza SA, Fang J, Gutterman DD, Wilcox DA, Bubolz AH, Li R, Suzuki M, Zhang DX (2010) TRPV4-mediated endothelial $\mathrm{Ca}^{2+}$ influx and vasodilation in response to shear stress. Am J Physiol Heart Circ Physiol 298: H466-H476. CrossRef Medline

Metea MR, Newman EA (2006) Glial cells dilate and constrict blood vessels: a mechanism of neurovascular coupling. J Neurosci 26:2862-2870. CrossRef Medline

Mulligan SJ, MacVicar BA (2004) Calcium transients in astrocyte endfeet cause cerebrovascular constrictions. Nature 431:195-199. CrossRef Medline

Nakahata K, Kinoshita H, Tokinaga Y, Ishida Y, Kimoto Y, Dojo M, Mizumoto K, Ogawa K, Hatano Y (2006) Vasodilation mediated by inward rectifier $\mathrm{K}+$ channels in cerebral microvessels of hypertensive and normotensive rats. Anesth Analg 102:571-576. CrossRef Medline

Niwa K, Kazama K, Younkin L, Younkin SG, Carlson GA, Iadecola C (2002) Cerebrovascular autoregulation is profoundly impaired in mice overexpressing amyloid precursor protein. Am J Physiol Heart Circ Physiol 283:H315-H323. CrossRef Medline

Plant TD, Strotmann R (2007) TRPV4: a multifunctional nonselective cation channel with complex regulation. In: TRP ion channel function in sensory transduction and cellular signaling cascades (Liedtke WB, Heller S, eds). Boca Raton, FL: CRC.

Shen Z, Lu Z, Chhatbar PY, O'Herron P, Kara P (2012) An artery-specific fluorescent dye for studying neurovascular coupling. Nat Methods 9:273276. CrossRef Medline
Simard M, Arcuino G, Takano T, Liu QS, Nedergaard M (2003) Signaling at the gliovascular interface. J Neurosci 23:9254-9262. Medline

Takano T, Han X, Deane R, Zlokovic B, Nedergaard M (2007) Two-photon imaging of astrocytic $\mathrm{Ca}^{2+}$ signaling and the microvasculature in experimental mice models of Alzheimer's disease. Ann N Y Acad Sci 1097:4050. CrossRef Medline

Thrane AS, Rangroo Thrane V, Zeppenfeld D, Lou N, Xu Q, Nagelhus EA, Nedergaard M (2012) General anesthesia selectively disrupts astrocyte calcium signaling in the awake mouse cortex. Proc Natl Acad Sci U S A 109:18974-18979. CrossRef Medline

Toth P, Rozsa B, Springo Z, Doczi T, Koller A (2011) Isolated human and rat cerebral arteries constrict to increases in flow: role of 20-HETE and TP receptors. J Cereb Blood Flow Metab 31:2096-2105. CrossRef Medline

Vriens J, Watanabe H, Janssens A, Droogmans G, Voets T, Nilius B (2004) Cell swelling, heat, and chemical agonists use distinct pathways for the activation of the cation channel TRPV4. Proc Natl Acad Sci U S A 101: 396-401. CrossRef Medline

Watanabe H, Vriens J, Prenen J, Droogmans G, Voets T, Nilius B (2003) Anandamide and arachidonic acid use epoxyeicosatrienoic acids to activate TRPV4 channels. Nature 424:434-438. CrossRef Medline

Zhang L, Papadopoulos P, Hamel E (2013) Endothelial TRPV4 channels mediate dilation of cerebral arteries: impairment and recovery in cerebrovascular pathologies related to Alzheimer's disease. Br J Pharmacol 170: 661-670. CrossRef Medline

Zonta M, Angulo MC, Gobbo S, Rosengarten B, Hossmann KA, Pozzan T, Carmignoto G (2003) Neuron-to-astrocyte signaling is central to the dynamic control of brain microcirculation. Nat Neurosci 6:43-50. Medline 